\title{
INTERPRÉTATION DE L'ARITHMÉTIQUE DANS CERTAINS GROUPES DE PERMUTATIONS AFFINES PAR MORCEAUX D'UN INTERVALLE
}

\author{
TUNA ALTINEL ET ALEXEY MURANOV
}

\begin{abstract}
The Arithmetic is interpreted in all the groups of Richard Thompson and Graham Higman, as well as in other groups of piecewise affine permutations of an interval which generalize the groups of Thompson and Higman. In particular, the elementary theories of all these groups are undecidable. Moreover, Thompson's group $F$ and some of its generalizations interpret the Arithmetic without parameters.
\end{abstract}

RÉsumÉ. L'Arithmétique est interprétée dans tous les groupes de Richard Thompson et de Graham Higman, aussi bien que dans d'autres groupes des permutations affines par morceaux d'un intervalle qui généralisent les groupes de Thompson et de Higman. En particulier, les théories élémentaires de tous ces groupes sont indécidables. De plus, le groupe $F$ de Thompson et certaines de ses généralisations interprètent l'Arithmétique sans paramètres.

\section{TABLE DES MATIÈRES}

1. Introduction $\underline{2}$

2. Généralités $\underline{4}$

2.1. Permutations et applications affines par morceaux $\underline{4}$

2.2. Groupes à l'étude $\underline{\underline{6}}$

2.3. Théories et modèles $\quad \underline{7}$

2.4. Décidabilité $\underline{9}$

3. Deux lemmes $\underline{10}$

4. Copies définissables de $\mathbb{Z} \imath \mathbb{Z}$

5. Interprétations de l'Arithmétique $\underline{17}$

6. Indécidabilité $\underline{22}$

7. Questions ouvertes

8. Appendice $\underline{26}$

Références $\quad \underline{27}$

Date: 26 octobre 2018.

2000 Mathematics Subject Classification. Principale 03C62; secondaire 20F65, 03D35.

Key words and phrases. Groupes de Thompson, groupe simple de présentation finie, théorie élémentaire, interprétation, arithmétique, indécidabilité héréditaire.

Le second auteur a été soutenu d'abord par une bourse Chateaubriand et après par FP6 Marie Curie Research Training Network in Model Theory and its Applications financé par la commission européenne sous le contrat numéro MRTN-CT-2004-512234 (MODNET). 


\section{INTRODUCTION}

Valery Bardakov et Vladimir Tolstykh [2] ont récemment montré que le groupe $F$ de Richard Thompson interprète l'Arithmétique. En d'autres termes, $F$ interprète la structure $(\mathbb{N},+, \times)$ par des formules du premier ordre avec paramètres. Dans ce travail nous généralisons ce résultat dans deux directions. D'un côté, dans les sections 3 et 4 nous généralisons l'approche de Bardakov et Tolstykh à la fabrication de l'Arithmétique à partir de $F$ et montrons qu'elle marche pour tous les groupes définis par Melanie Stein dans [33], dont tous les trois groupes de Thompson et tous les groupes de Graham Higman [14]. D'un autre côté, dans la section 5 nous démontrons que le groupe $F$ et certaines de ses généralisations interprètent l'Arithmétique sans paramètres. (La différence entre avec et sans paramètres sera expliquée dans la section 2.3.)

La théorie élémentaire de l'Arithmétique $(\mathbb{N},+, \times)$ est notoire pour sa complexité depuis les théorèmes d'incomplétude de Gödel [13]. L'une des significations de l'interprétabilité de l'Arithmétique dans une structure de signature finie est ce qu'elle entraîne l'indécidabilité héréditaire de la théorie élémentaire de cette structure. (Une théorie de signature finie est dite héréditairement indécidable si toute sous-théorie de la même signature est indécidable, voir [34, §3].) Grâce à des travaux d'Andrzej Mostowski, de Raphael Robinson, et d'Alfred Tarski [19, 20, 34], il est bien connu que la théorie élémentaire de l'Arithmétique est héréditairement indécidable. Il est également bien connu aux spécialistes que si une structure $N$ de signature finie interprète avec paramètres une autre structure $M$ de signature finie et dont la théorie élémentaire est héréditairement indécidable, alors la théorie élémentaire de $N$ l'est aussi. ${ }^{1}$ Ainsi Bardakov et Tolstykh ont démontré que la théorie élémentaire de $F$ est héréditairement indécidable, et donc une partie de la question numéro 4.16 par Mark Sapir dans [36] est résolue. Par le même raisonnement, les théories élémentaires de tous les groupes que nous étudions dans ce travail sont héréditairement indécidables.

Pour confort du lecteur, nous présentons dans la section 6 notre version d'une preuve, basée elle aussi sur un résultat de Mostowski, Robinson, et Tarski [19, théorème 9], que si une structure $S$ de signature finie interprète l'Arithmétique avec paramètres, alors la théorie élémentaire de $S$ est héréditairement indécidable.

Les groupes qui font l'objet de notre étude apparaissent naturellement comme des généralisations des trois groupes définis par Thompson en 1965 et habituellement notés $F, T$, et $V{ }^{2}$ Les groupes de Thompson sont exposés en détail dans $[3, \underline{8}]$. Tous les trois sont infinis et de présentation finie. Le groupe $V$ était le premier exemple connu d'un groupe simple infini de présentation finie. Le groupe $T$ est simple aussi. Le groupe $F$ se plonge dans $T$, et $T$ se plonge dans $V$. Le groupe $V$ a été généralisé par Higman [14] en une série de groupes $G_{n, r}, n=2,3,4, \ldots, r=1,2,3, \ldots$, de présentation finie, où $G_{2,1} \cong V$. Le groupe $G_{n, r}$ de Higman est simple lorsque $n$ est pair ;

\footnotetext{
${ }^{1}$ Dans [2] les auteurs affirment ce fait avec référence à [12]. Nous démontrons ce fait comme le lemme 6.2 .

${ }^{2}$ D'autres lettres ont aussi été utilisées pour noter ces groupes (voir [8]). Il est assez habituel, par exemple, de noter le groupe $V$ par $G$.
} 
lorsque $n$ est impair, le sous-groupe dérivé $\left[G_{n, r}, G_{n, r}\right]$ est simple et d'indice 2 dans $G_{n, r}$. Kenneth Brown [7, section 4] a généralisé de la même façon les groupes $F$ et $T$.

Les groupes de Thompson ont des représentations par des permutations affines par morceaux d'un intervalle, où le groupe $F$ est représenté par des homéomorphismes par rapport à la topologie habituelle, et $T$ est représenté par des homéomorphismes par rapport à la topologie de cercle. Stein [33] a étudié trois familles de groupes de telles permutations qui généralisent respectivement les trois groupes de Thompson. Afin d'énoncer nos résultats principaux, nous réviserons ici les définitions de ces familles.

Soient $r$ un nombre réel positif et $\Lambda$ un sous-groupe du groupe multiplicatif $\mathbb{R}_{+}^{*}$ des nombres réels positifs. Soit $A$ un sous-groupe additif de $\mathbb{R}$ contenant $r$ et invariant sous l'action de $\Lambda$ par multiplication. Alors définissons $\mathcal{V}(r, \Lambda, A)$ le groupe de toutes les bijections $x:[0 ; r[\rightarrow[0 ; r[$ qui satisfont les conditions suivantes :

(1) $x$ est affine par morceaux avec un nombre fini des coupures et des singularités ;

(2) $x$ est continue à droite en tout point (au sens habituel);

(3) la pente de chaque partie affine de $x$ est dans $\Lambda$;

(4) tous points de coupure et de singularité de $x$, ainsi que leurs images, sont dans $A$.

La famille des groupes $\mathcal{V}(r, \Lambda, A)$ contiens tous les groupes de Higman : pour tout $n=2,3, \ldots$ et tout $r=1,2, \ldots$,

$$
G_{n, r} \cong \mathcal{V}\left(r,\langle n\rangle, \mathbb{Z}\left[\frac{1}{n}\right]\right) .
$$

Définissons les sous-groupes $\mathcal{F}(r, \Lambda, A)$ et $\mathcal{T}(r, \Lambda, A)$ de $\mathcal{V}(r, \Lambda, A)$ comme suit :

- $\mathcal{F}(r, \Lambda, A)$ est le sous-groupe de tous les éléments de $\mathcal{V}(r, \Lambda, A)$ continus par rapport à la topologie habituelle de $[0 ; r[$,

- $\mathcal{T}(r, \Lambda, A)$ est le sous-groupe de tous les éléments de $\mathcal{V}(r, \Lambda, A)$ continus par rapport à la topologie de cercle sur $[0 ; r[$

(où la topologie de cercle sur [0; $r$ [ est la topologie induite par l'identification naturelle de $[0 ; r$ avec le quotient topologique $[0 ; r] /\{0, r\})$. Les groupes $F$, $T$, et $V$ de Thompson sont isomorphes à $\mathcal{F}(1,\langle 2\rangle, \mathbb{Z}[1 / 2]), \mathcal{T}(1,\langle 2\rangle, \mathbb{Z}[1 / 2])$, et $\mathcal{V}(1,\langle 2\rangle, \mathbb{Z}[1 / 2])$, respectivement. Des groupes de la forme $\mathcal{F}(r, \Lambda, A)$ ont été étudiés déjà par Robert Bieri et Ralph Strebel dans [4] (non publié).

Pour le reste nous supposons toujours que $\Lambda \neq\{1\}$.

Théorème A. Si $G$ est un sous-groupe de $\mathcal{V}\left(r, \mathbb{R}_{+}^{*}, \mathbb{R}\right)$ tel que

$$
G \cap \mathcal{F}\left(r, \mathbb{R}_{+}^{*}, \mathbb{R}\right)=\mathcal{F}(r, \Lambda, A),
$$

alors $G$ interprète l'Arithmétique $(\mathbb{N},+, \times)$ avec paramètres.

Théorème B. Si $\Lambda$ est cyclique, alors $\mathcal{F}(r, \Lambda, A)$ interprète l'Arithmétique sans paramètres.

Théorème C. Si $G$ est un groupe comme dans le théorème A, alors la théorie élémentaire de $G$ est héréditairement indécidable. 
En particulier, tous les groupes de Thompson et de Higman interprètent l'Arithmétique avec paramètres, alors que le groupe $F$ de Thompson l'interprète aussi sans paramètres, et les théories élémentaires de tous ces groupes sont héréditairement indécidables.

Les théorèmes $\mathrm{A}$ et $\mathrm{B}$ sont démontrés dans la section $\underline{5}$. À notre connaissance, l'interprétation construite dans la preuve du théorème $\mathrm{B}$ est entièrement originale. Le théorème $\mathrm{C}$ est démontré dans la section 6 comme un corollaire du théorème $\mathrm{A}$. Dans l'appendice, nous démontrons que tout élément du sous-groupe dérivé de $\mathcal{F}(r, \Lambda, A)$ est le produit de deux commutateurs, et donc que le sous-groupe dérivé est définissable dans $\mathcal{F}(r, \Lambda, A)$.

L'idée principale de la preuve du théorème A est, comme dans [2], de trouver dans $G$ un sous-groupe définissable isomorphe au produit en couronne restreint $\mathbb{Z} \imath \mathbb{Z}$, parce qu'il est connu que ce dernier groupe interprète l'Arithmétique. Remarquons que, en contraste avec $\mathbb{Z} Z \mathbb{Z}$ et avec les groupes à l'étude, ni les groupes abéliens, ni les groupes virtuellement abéliens, ni les groupes libres, ni les groupes hyperboliques sans torsion ne peuvent interpréter l'Arithmétique car leurs théories élémentaires sont tous stables, alors que la théorie élémentaire de toute structure qui interprète l'Arithmétique est «fortement » instable. La stabilité est une notion fondamentale de la théorie des modèles, à l'étude de laquelle $[24,25]$ sont d'excellentes introductions. Les meilleures sources pour l'étude des groupes stables, c'està-dire des groupes dont les théories élémentaires sont stables, ce sont, à notre avis, $[26,27,35]$. Une démonstration de la stabilité des groupes abéliens se trouve dans [28, théorème 3.1$]$. Tout groupe hyperbolique non élémentaire sans torsion est stable selon un résultat récent de Zlil Sela [32].

Un sous-groupe définissable de $F$ isomorphe à $\mathbb{Z} \geq \mathbb{Z}$ a été choisi par Bardakov et Tolstykh [2] comme suit. Soient $x_{0}$ et $x_{1}$ les générateurs « standards » de $F$, et soient $a=x_{0}^{2}, b=x_{1} x_{0}^{-1} x_{1}^{-1} x_{0}$ (voir la figure 1 ). On peut vérifier sans grande difficulté que $\langle a, b\rangle=\langle b\rangle\langle\langle a\rangle \cong \mathbb{Z} \imath \mathbb{Z}$. Le centralisateur de $x_{0}$ dans $F$ coïncide avec le sous-groupe engendré par $x_{0}$. En conséquence, le sous-groupe $\langle a\rangle$ est définissable dans $F$ par une formule avec le paramètre $x_{0}$. Puis il est montré que le centralisateur du sous-ensemble $\left\{a^{-k} b a^{k} \mid k \in \mathbb{Z}\right\}$ coïncide avec le sous-groupe $\left\langle a^{-k} b a^{k} \mid k \in \mathbb{Z}\right\rangle$. Comme le sous-ensemble $\left\{a^{-k} b a^{k} \mid k \in \mathbb{Z}\right\}$ est clairement définissable avec les paramètres $x_{0}$ et $x_{1}$, il en est de même pour le sous-groupe $\left\langle a^{-k} b a^{k} \mid k \in \mathbb{Z}\right\rangle$. Donc le sous-groupe $\langle a, b\rangle \cong \mathbb{Z} \backslash \mathbb{Z}$ est définissable dans $F$ avec paramètres. Dans la preuve du théorème $\mathrm{A}$, nous suivons une approche similaire pour le groupe $\mathcal{F}(r, \Lambda, A)$.

\section{GÉNÉRALités}

Dans cette section, nous présentons des définitions de base et quelques faits élémentaires.

\subsection{Permutations et applications affines par morceaux.}

Définition. Une bijection d'un ensemble sur lui-même est dite une permutation de cet ensemble. Une application $f$ est dite de permuter un ensemble $S$ si la restriction $\left.f\right|_{S}$ est une permutation de $S$. 

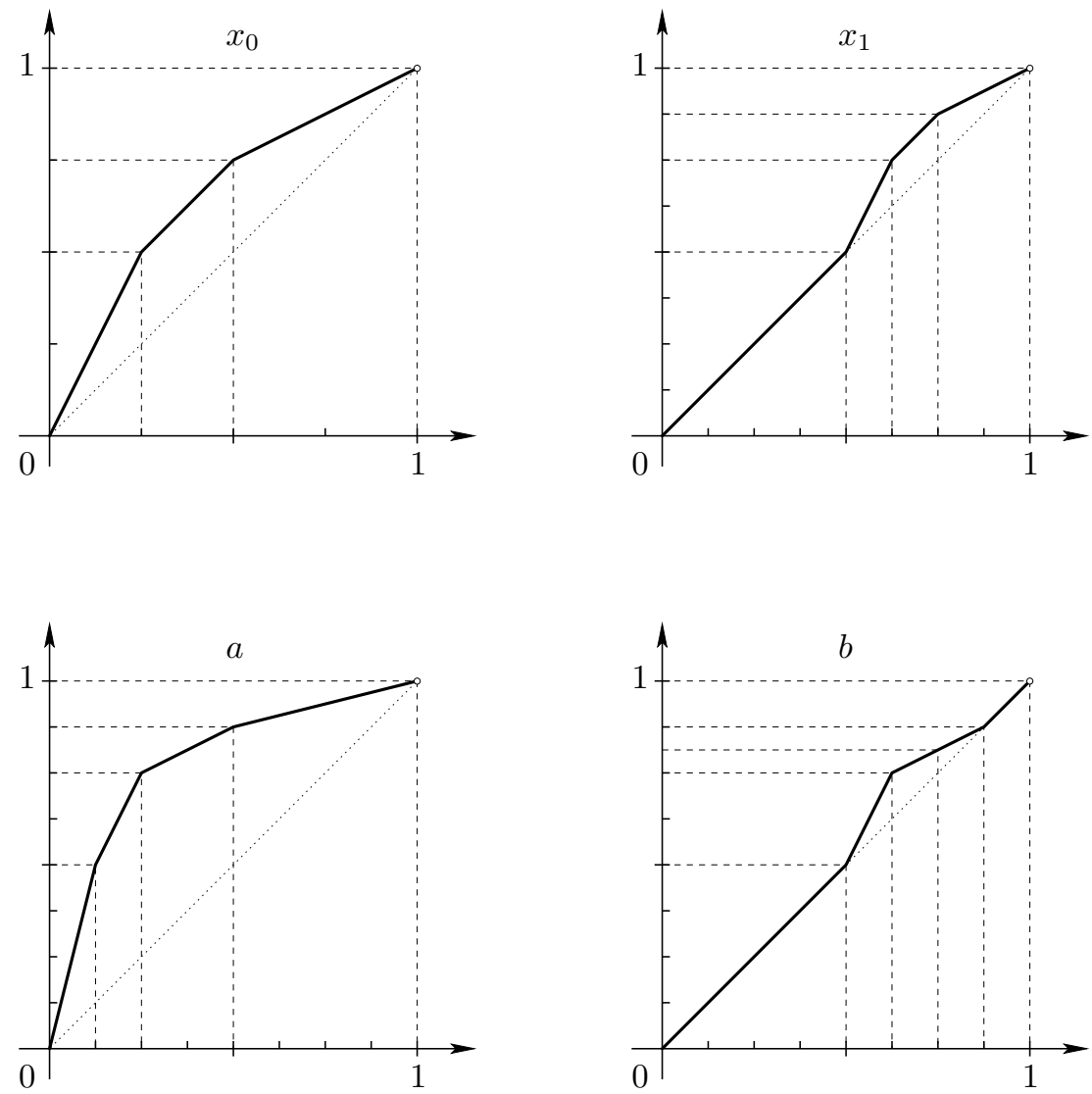

Fig. 1. Applications $x_{0}, x_{1}, a=x_{0}^{2}$, et $b=x_{1} x_{0}^{-1} x_{1}^{-1} x_{0}$.

Définition. Soient $S$ un ensemble et $f$ une bijection de $S$ sur lui-même. Appelons support de $f$, noté $\operatorname{Supp}(f)$, le complément dans $S$ de l'ensemble des points fixes de $f$, noté $\operatorname{Fix}(f)$.

Par coutume, dans le contexte de l'étude des groupes de Thompson et de Higman toutes les applications agissent à droite. Nous adoptons la même convention dans cet article; par exemple : $(\alpha)(x y)=((\alpha) x) y$ si $x$ et $y$ sont des permutations d'un ensemble $S$, et $\alpha \in S$.

Nous allons écrire $X^{f}$, ou parfois $(X) f$, pour noter l'image de l'ensemble $X$ sous l'application $f$.

Les lemmes 2.1, 2.2, et 2.3 sont évidents :

Lemme 2.1. Deux permutations d'un même ensemble commutent si leurs supports sont disjoints.

Lemme 2.2. Soient $f$ et $g$ deux permutations d'un même ensemble. Alors

$$
\begin{aligned}
\operatorname{Fix}\left(g^{-1} f g\right) & =(\operatorname{Fix}(f)) g, \\
\operatorname{Supp}\left(g^{-1} f g\right) & =(\operatorname{Supp}(f)) g .
\end{aligned}
$$

Lemme 2.3. Soient $f$ et $g$ deux permutations d'un même ensemble qui commutent. Alors g permute chacun des ensembles $\operatorname{Fix}(f)$ et $\operatorname{Supp}(f)$. 
Lemme 2.4. Soient I un intervalle dans $\mathbb{R}, f$ une permutation croissante de $I$, et $n \in \mathbb{Z} \backslash\{0\}$. Alors $\operatorname{Supp}\left(f^{n}\right)=\operatorname{Supp}(f)$.

Démonstration. Clairement $\operatorname{Fix}(f) \subset \operatorname{Fix}\left(f^{n}\right)$ et $\operatorname{Supp}(f) \supset \operatorname{Supp}\left(f^{n}\right)$. Considérons $\alpha \in \operatorname{Supp}(f)$ arbitrairement choisi. Sans perte de généralité, supposons que $(\alpha) f>\alpha$. Alors

$$
\alpha<(\alpha) f<(\alpha) f^{2}<\cdots<(\alpha) f^{n},
$$

et donc $\alpha \in \operatorname{Supp}\left(f^{n}\right)$.

Lemme 2.5. Soient I un intervalle compact dans $\mathbb{R}, f$ une permutation croissante de $I$, et $\alpha \in I$. Alors

$$
\lim _{n \rightarrow+\infty}(\alpha) f^{n} \in \operatorname{Fix}(f) .
$$

Démonstration. Comme $f$ préserve l'ordre, la suite $\left((\alpha) f^{n}\right)_{n=0,1, \ldots}$ est monotone, et donc la limite existe et elle appartient à $I$. Soit $\beta=\lim _{n \rightarrow+\infty}(\alpha) f^{n}$, alors $(\beta) f=\beta$ par continuité.

Définition. Soient $f$ une application et $\alpha$ un nombre réel. Disons que $f$ est affine $\grave{a}$ droite de $\alpha$ s'il existe $\beta>\alpha$ tel que la restriction $\left.f\right|_{] \alpha ; \beta[}$ soit une application affine $] \alpha ; \beta[\rightarrow \mathbb{R}$. Disons que $f$ est affine à gauche de $\alpha$ s'il existe $\beta<\alpha$ tel que $\left.f\right|_{] \beta ; \alpha[}$ soit une application affine $] \beta ; \alpha[\rightarrow \mathbb{R}$.

Définition. Pour tous $\alpha, \beta \in \mathbb{R}$ tels que $\alpha<\beta$, et pour toute application $f$ telle que $\left.f\right|_{] \alpha ; \beta[}$ soit une application affine $] \alpha ; \beta[\rightarrow \mathbb{R}$, nous noterons la pente de $\left.f\right|_{] \alpha ; \beta[} \operatorname{par}(\alpha) f^{\prime+}$ et également par $(\beta) f^{\prime-}$. Disons que $(\alpha) f^{\prime+}$ est la pente de $f \grave{a}$ droite de $\alpha$, et que $(\beta) f^{\prime-}$ est la pente de $f$ à gauche de $\beta$.

On démontre le lemme suivant de la même façon qu'on détermine la fonction dérivée d'une fonction composée.

Lemme 2.6. Soient $f$ et $g$ deux applications des sous-ensembles de $\mathbb{R}$ vers $\mathbb{R}$, et $\alpha \in \mathbb{R}$.

(1) Si $f$ est affine à droite de $\alpha$ avec $(\alpha) f^{\prime+}>0, f$ est continue en $\alpha \grave{a}$ droite, et $g$ est affine à droite de $(\alpha) f$, alors

$$
(\alpha)(f g)^{\prime+}=(\alpha) f^{\prime+} \cdot((\alpha) f) g^{\prime+} .
$$

(2) Si $f$ est affine à gauche de $\alpha$ avec $(\alpha) f^{\prime-}>0, f$ est continue en $\alpha$ $\grave{a}$ gauche, et $g$ est affine à gauche de $(\alpha) f$, alors

$$
(\alpha)(f g)^{\prime-}=(\alpha) f^{\prime-} \cdot((\alpha) f) g^{\prime-} .
$$

2.2. Groupes à l'étude. Comme dans l'introduction, choisissons un nombre réel positif $r$, un sous-groupe $\Lambda$ du groupe multiplicatif $\mathbb{R}_{+}^{*}$, et un sousmodule $A$ du $\Lambda$-module $\mathbb{R}$ tel que $r \in A$. Appelons un tel triple $(r, \Lambda, A) a d$ missible. Pour un triple $(r, \Lambda, A)$ admissible, définissons les groupes $\mathcal{F}(r, \Lambda, A)$, $\mathcal{T}(r, \Lambda, A)$, et $\mathcal{V}(r, \Lambda, A)$ comme dans l'introduction. Nous allons traiter les groupes $F, T$, et $V$ de Thompson comme des cas particuliers, donc nous posons

$$
F=\mathcal{F}\left(1,\langle 2\rangle, \mathbb{Z}\left[\frac{1}{2}\right]\right), \quad T=\mathcal{T}\left(1,\langle 2\rangle, \mathbb{Z}\left[\frac{1}{2}\right]\right), \quad V=\mathcal{V}\left(1,\langle 2\rangle, \mathbb{Z}\left[\frac{1}{2}\right]\right) .
$$

Remarque 2.7. Tout élément de $\mathcal{F}\left(r, \mathbb{R}_{+}^{*}, \mathbb{R}\right)$ s'étend d'une façon unique à un homéomorphisme $[0 ; r] \rightarrow[0 ; r]$ par rapport à la topologie habituelle. 
Remarque 2.8. La conjugaison des éléments de $\mathcal{V}\left(1, \mathbb{R}_{+}^{*}, \mathbb{R}\right)$ par l'application linéaire de multiplication par $r$ est un isomorphisme entre $\mathcal{V}\left(1, \mathbb{R}_{+}^{*}, \mathbb{R}\right)$ et $\mathcal{V}\left(r, \mathbb{R}_{+}^{*}, \mathbb{R}\right)$, qui envoie $\mathcal{V}\left(1, \Lambda, A r^{-1}\right)$ sur $\mathcal{V}(r, \Lambda, A), \mathcal{T}\left(1, \Lambda, A r^{-1}\right)$ sur $\mathcal{T}(r, \Lambda, A)$, et $\mathcal{F}\left(1, \Lambda, A r^{-1}\right)$ sur $\mathcal{F}(r, \Lambda, A)$, où $A r^{-1}=\{\alpha / r \mid \alpha \in A\}$.

Il est commode de distinguer dans $\mathcal{F}(r, \Lambda, A)$ les sous-semigroupes suivants :

- notons $\mathcal{F}^{\uparrow}(r, \Lambda, A)$ le semigroupe de tous les éléments $x \in \mathcal{F}(r, \Lambda, A)$ tels que $(\alpha) x \geq \alpha$ pour tout $\alpha \in[0 ; r[$, et

- notons $\mathcal{F}^{\downarrow}(r, \Lambda, A)$ le semigroupe de tous les $x \in \mathcal{F}(r, \Lambda, A)$ tels que $(\alpha) x \leq \alpha$ pour tout $\alpha \in[0 ; r[$.

Introduisons d'autres notations utiles : soit $S \subset[0 ; r[$, alors

$$
\begin{aligned}
\mathcal{F}_{S}(r, \Lambda, A) & =\{x \in \mathcal{F}(r, \Lambda, A) \mid \operatorname{Supp}(x) \subset S\}, \\
\mathcal{T}_{S}(r, \Lambda, A) & =\{x \in \mathcal{T}(r, \Lambda, A) \mid \operatorname{Supp}(x) \subset S\}, \\
\mathcal{V}_{S}(r, \Lambda, A) & =\{x \in \mathcal{V}(r, \Lambda, A) \mid \operatorname{Supp}(x) \subset S\} .
\end{aligned}
$$

On définit de même les semigroupes $\mathcal{F}_{S}^{\uparrow}(r, \Lambda, A)$ et $\mathcal{F}_{S}^{\downarrow}(r, \Lambda, A)$.

Pour le reste de cet article, nous fixons $(r, \Lambda, A)$, et de plus, nous supposons que $\Lambda$ n'est pas trivial : $\Lambda \neq\{1\}$. Afin de simplifier la notation, nous posons :

$$
\mathcal{F}=\mathcal{F}(r, \Lambda, A), \quad \mathcal{T}=\mathcal{T}(r, \Lambda, A), \quad \mathcal{V}=\mathcal{V}(r, \Lambda, A) .
$$

Également, nous allons écrire $\mathcal{F}^{\uparrow}$ au lieu de $\mathcal{F}^{\uparrow}(r, \Lambda, A)$, etc.

2.3. Théories et modèles. Dans cet article nous parlons des structures au sens de la théorie des modèles (ou au sens de l'algèbre universelle, à quelques distinctions linguistiques près). Lorsque les termes " formule ", « énoncé », et « théorie » sont utilisés dans le sens formel, ils signifient toujours des formules, des énoncés, et des théories du premier ordre. Les termes 《 théorie », «théorie élémentaire », et « théorie du premier ordre » seront utilisés comme des synonymes. «Un modèle » et « une structure » seront généralement synonymes aussi. Sauf indication contraire, les formules sont sans paramètres.

Une structure $M$ de signature $\Sigma$, dite aussi $\Sigma$-structure, est un modèle d'un ensemble d'énoncés $S$, ce qui est noté $M \models S$, si $M$ satisfait tout énoncé $\alpha$ de $S$, ce qui est noté $M \models \alpha$. Un énoncé $\alpha$ est dit une conséquence d'un ensemble d'énoncés $S$ dans une signature $\Sigma$, ce qui est noté $S \vdash_{\Sigma} \alpha$ ou $S \vdash \alpha$ au cas où $\Sigma$ est bien comprise, si tout $\Sigma$-modèle de $S$ est aussi un modèle de $\alpha$. Un ensemble d'énoncés est consistant dans une signature $\Sigma$ s'il a un $\Sigma$-modèle. ${ }^{3}$ Un ensemble d'énoncés est déductivement clos dans une signature $\Sigma$ s'il contient toutes ses conséquences dans $\Sigma$. Une théorie de signature $\Sigma$, dite aussi $\Sigma$-théorie, est un ensemble d'énoncés consistant et déductivement clos dans $\Sigma$. Si $T$ est une $\Sigma$-théorie, alors $T \vdash_{\Sigma} \alpha$ équivaut à $\alpha \in T$. La théorie d'une structure $M$, notée $\operatorname{Th}(M)$, est l'ensemble de tous les énoncés dans la signature de $M$ satisfaits par $M$. Une théorie est dite complète si elle est la théorie d'une structure. La classe de tous les $\Sigma$-modèles d'un ensemble de $\Sigma$-énoncés $S$ est notée $\operatorname{Mod}_{\Sigma}(S)$.

\footnotetext{
${ }^{3}$ La signature $\Sigma$ dans cette définition est d'importance mineure sauf au cas où $S$ a un modèle vide, ce qui signifierait en particulier qu'aucun élément de $S$ ne comporte de symboles de constante.
} 
Nous allons utiliser implicitement le théorème de compacité, qui assure qu'une conséquence d'un ensemble d'énoncés est toujours une conséquence, dans la même signature, d'une de ses parties finies. Nous recommandons un des ouvrages $[15, \underline{16}, 25, \underline{30}$ ] pour références sur des résultats généraux de la théorie de modèles.

Si $M$ est une structure, un ensemble définissable dans $M$ est en général une partie de $M^{n}$, où $n \in \mathbb{N}$, définissable par une formule du premier ordre dans la signature de $M$, et éventuellement avec des paramètres extraits de $M$. Les paramètres sont de nouveaux symboles de constante rajoutés dans le langage et interprétés par des éléments de $M$. (D'habitude, pour nommer un élément $a \in M$, on utilise $a$ lui-même comme un paramètre.) Par exemple, dans un groupe, le centralisateur de tout élément $g$ est définissable par la formule $\phi(x)=\ulcorner g x=x g\urcorner$ avec $g$ comme un paramètre, mais en général il n'y a aucune raison pour que ce centralisateur soit définissable par une formule sans paramètres dans la signature de groupe pur, qui ne comporte qu'un seul symbole de fonction binaire $\ulcorner$.$\urcorner pour noter l'opération$ de groupe $(x, y) \mapsto x \cdot y$. Pour préciser, un ensemble est dit « définissable avec » ou «sans paramètres » selon si des paramètres sont permis ou pas dans sa définition. Par contre, nous n'allons pas préciser la structure dans laquelle un ensemble donné sera définissable, sauf s'il y a plusieurs choix également naturels dans le contexte. Bien entendu, on peut parler aussi de la définissabilité des relations et des opérations.

Si $f$ est une application $A \rightarrow B$, et que $n$ est un entier positif, nous notons $f^{\underline{n}}$ l'application $A^{n} \rightarrow B^{n}$ induite par $f$. Nous allons légèrement abuser la notation en supposant que si l'ensemble de départ de $f$ est une partie de $A^{m}$, alors l'ensemble de départ de $f \underline{n}$ est identifié naturellement avec une partie de $A^{m n}$. Nous appelons la $f$-préimage d'un ensemble donné sa préimage sous $f^{\underline{n}}$ au cas où le choix de $n$ est évident (donc pas nécessairement sous $f$ elle-même).

Considérons deux structures, $M$ de signature $\Sigma$ et $N$ de signature $\Gamma$.

Définition. Nous appelons une interprétation avec paramètres de $M$ dans $N$ une paire $(n, f)$ où $n \in \mathbb{N}$ et où $f$ est une application surjective d'une partie de $N^{n}$ sur $M$, telle que pour tout ensemble $X$ définissable dans $M$ sans paramètres, la $f$-préimage de $X$ soit définissable (dans $N$ ) avec (éventuellement) paramètres. Une interprétation $(n, f)$ avec paramètres est dite une interprétation sans paramètres si la $f$-préimage de tout ensemble définissable sans paramètres est, elle aussi, définissable sans paramètres.

Voir [15, chapitre 5] pour des explications détaillées de l'interprétabilité et des notions liées.

Dans ce qui suit, conformément à l'usage en théorie des modèles, les termes « définissable », « 0-définissable », « interprétation », et « 0-interprétation » seront utilisés pour noter 《 définissable avec paramètres », 《 définissable sans paramètres », « interprétation avec paramètres », et « interprétation sans paramètres », respectivement. Aussi, comme dans notre cas la valeur de $n$ pour une interprétation $(n, f)$ à l'étude sera souvent soit bien comprise, soit peu importante, nous allons simplifier la notation et appeler $f$ elle-même une interprétation. Pour noter que $(n, f)$ est une interprétation 
de $M$ dans $N$, nous allons écrire soit $(n, f): M \rightsquigarrow N$, soit $f: M \rightsquigarrow N .^{4}$ Pour noter que $f$ est une 0 -interprétation de $M$ dans $N$, nous écrivons $f: M \stackrel{\infty}{\rightsquigarrow} N$. Pour noter que $M$ est interprétable ou 0-interprétable dans $N$, nous écrivons $M \rightsquigarrow N$ ou $M \stackrel{\wp}{\rightsquigarrow}$, respectivement.

Remarque 2.9. Si $f: M \rightsquigarrow N$, alors la $f$-préimage de tout ensemble définissable dans $M$ est, elle aussi, définissable (dans $N$ ).

Remarque 2.10. Soient $n \in \mathbb{N}$ et $B \subset N^{n}$. Alors une application surjective $f$ de $B$ sur $M$ est une interprétation de $M$ dans $N$ si et seulement si

(1) l'ensemble de départ $B$ est définissable,

(2) la relation d'équivalence sur $B$ induite par $f$ (le noyau de $f$ ) est définissable, et

(3) pour toute relation, opération, et constante de la structure $M$ (nommée par un symbole de $\Sigma$ ), la $f$-préimage de son graphe est définissable.

L'application $f$ est une 0-interprétation si et seulement si tous ces ensembles sont 0-définissables.

Remarque 2.11. Si $L, M$, et $N$ sont trois structures, et que $(m, f): L \rightsquigarrow M$ et $(n, g): M \rightsquigarrow N$, alors $\left(m n, g^{\underline{m}} f\right): L \rightsquigarrow N$. Si de plus $f$ et $g$ sont 0 interprétations, alors $g^{\underline{m}} f$ l'est aussi.

Définition (voir [1] et [15, section 5.4(c)]). Deux structures $M$ et $N$ sont dites bi-interprétables s'il existe deux interprétations $(m, f): M \rightsquigarrow N$ et $(n, g): N \rightsquigarrow M$ telles que l'application $g^{\underline{m}} f$ soit définissable dans $M$, et que $f^{\underline{n}} g$ soit définissable dans $N$. Les interprétations $(m, f)$ et $(n, g)$ dans ce cas sont dites bi-interprétations.

2.4. Décidabilité. Soit $A$ un ensemble fini vu comme un alphabet, et notons $A^{*}$ l'ensemble de tous les mots finis dans $A$. Nous appelons un ensemble $X \subset A^{*}$ récursif ou bien décidable s'il existe un algorithme qui pour toute donnée $w \in A^{*}$ répond à la question si $w \in X$. Une application $f: X \rightarrow A^{*}$ est dite calculable s'il existe un algorithme qui calcule $f(w)$ pour toute donnée $w \in X$, et qui ne s'arrête jamais pour toute donnée $w \notin X$.

Normalement on dit qu'un ensemble est récursif ou non récursif, tandis que une théorie est décidable ou indécidable.

Dans le reste de cette section, soit $\Sigma$ une signature finie arbitraire.

Définition. Une $\Sigma$-théorie $T$ est dite essentiellement indécidable si toute $\Sigma$-théorie contenant $T$ est indécidable.

Remarque 2.12. Si $T$ est une théorie de signature finie, et que une partie de $T$ est une théorie essentiellement indécidable (de signature éventuellement plus petite), alors $T$ est indécidable, et même essentiellement indécidable.

Définition. Une $\Sigma$-théorie $T$ est dite héréditairement indécidable si toute $\Sigma$-sous-théorie de $T$ est indécidable.

\footnotetext{
${ }^{4}$ La flèche ici se dirige dans le sens inverse de la notation de [1].
} 
Lemme 2.13 ([34, théorème 6$])$. Si $T$ est une théorie de signature finie, et que $T$ a une sous-théorie (de signature éventuellement plus petite) essentiellement indécidable et finiment axiomatisée, alors $T$ est héréditairement indécidable.

Démonstration. Notons $\Sigma$ la signature de $T$. Soit $S$ une sous-théorie de $T$ qui est essentiellement indécidable et finiment axiomatisée. Choisissons un énoncé $\theta \in S$ qui axiomatise $S$.

Soit par l'absurde $U$ une $\Sigma$-sous-théorie de $T$ qui est décidable. Soit $R$ la $\Sigma$-théorie axiomatisée (engendrée) par $U \cup S$. Alors

$$
R=\left\{\alpha \mid U, \theta \vdash_{\Sigma} \alpha\right\}=\{\alpha \mid\ulcorner\theta \rightarrow \alpha\urcorner \in U\},
$$

et donc $R$ est décidable puisque $U$ l'est. Cela contredit l'indécidabilité essentielle de $S$ (voir la remarque 2.12).

\section{Deux LEMmes}

Dans cette section, nous démontrerons deux lemmes techniques à propos des homéomorphismes affines par morceaux d'un intervalle. Ces lemmes seront essentiels pour la preuve du théorème $\mathrm{A}$, à savoir, pour démontrer que certains centralisateurs sont préservés en passant de $\mathcal{F}$ à $\mathcal{V}$, et ainsi pour pouvoir passer d'une interprétation de l'Arithmétique dans $\mathcal{F}$ à ses interprétations dans $\mathcal{V}$ et dans $\mathcal{T}$.

Lemme 3.1. Soit $r \in \mathbb{R}_{+}^{*}$. Soit $z$ un homéomorphisme $[0 ; r[\rightarrow[0 ; r[$ tel que $(\alpha) z>\alpha$ pour tout $\alpha \in] 0 ; r[$. Soit $f$ une permutation de $[0 ; r[$ telle que :

(1) $f$ commute avec $z$,

(2) $f$ soit continue (à droite) en 0 ,

(3) $f$ ait un nombre fini des points de discontinuité.

Alors $f$ est continue (par rapport à la topologie habituelle).

Démonstration. Supposons que $f$ ne soit pas continue. Alors soit $\alpha$ le plus petit élément de $[0 ; r$ [ où $f$ n'est pas continue. Comme $f$ est continue en 0 , $\alpha \in] 0 ; r\left[\right.$. Donc $(\alpha) z^{-1}<\alpha$ et $f$ est continue en $(\alpha) z^{-1}$. On conclut que $f$ est continue en $\alpha$ parce que $f=z^{-1} f z$, où $z^{-1}$ et $z$ sont continues partout, et $f$ est continue en $(\alpha) z^{-1}$. Cela donne une contradiction.

Lemme 3.2. Soit $r \in \mathbb{R}_{+}^{*}$. Soit $Z$ un ensemble d'homéomorphismes $[0 ; r[\rightarrow$ $[0 ; r[$ tel que :

(1) $(\alpha) z \geq \alpha$ pour tout $z \in Z$ et tout $\alpha \in[0 ; r[$,

(2) $\operatorname{Supp}(z)$ soit un intervalle pour tout $z \in Z$,

(3) $\bigcup_{z \in Z} \operatorname{Supp}(z)$ soit dense dans $] 0 ; r[$.

Soit $f$ une permutation de $[0 ; r[$ telle que :

(1) $f$ commute avec tout $z \in Z$,

(2) $f$ soit continue à droite en tout point de $[0 ; r[$,

(3) $f$ ait un nombre fini des points de discontinuité.

Alors $f$ est continue. 
Démonstration. Démontrons d'abord que $f$ est croissante sur chacun de ses intervalles de continuité.

Disons qu'un intervalle $I$ est fermé à droite si sup $I \in I$, et qu'il est fermé $\grave{a}$ gauche si inf $I \in I$. Si un intervalle n'est pas fermé à droite ou gauche, disons qu'il $y$ est ouvert.

Comme le nombre de points de discontinuité de $f$ est fini, le nombre de ses intervalles de continuité maximaux est fini aussi. Comme $f$ est continue à droite partout dans son ensemble de départ $[0 ; r[$, tout intervalle de continuité maximal est fermé à gauche et ouvert à droite. Alors l'image sous $f$ de tout intervalle $I$ de continuité maximal est fermé à gauche si $f$ est croissante sur $I$, et fermé à droite si $f$ est décroissante sur $I$.

Évidemment $[0 ; r[$ est la réunion disjointe des images des intervalles de continuité maximaux de $f$. Comme toute telle image est un intervalle soit ouvert à droite soit ouvert à gauche, la seule possibilité est qu'ils sont tous fermés à gauche et ouverts à droite. Donc $f$ est croissante sur chacun de ses intervalles de continuité.

Supposons maintenant que $f$ ne soit pas continue.

D'après le lemme 2.3, pour tout $z \in Z, f$ permute l'ensemble $\operatorname{Supp}(z)$. Démontrons que $f$ est continue sur chacun de ces intervalles. Soit par l'absurde $z \in Z$ tel que $f$ ne soit pas continue $\operatorname{sur} \operatorname{Supp}(z)$. Soit $\gamma$ le plus petit élément de $\operatorname{Supp}(z)$ où $f$ n'est pas continue. Alors $f$ est continue en $\gamma$ parce que $f=z^{-1} f z$, où $z$ est un homéomorphisme, et $f$ est continue en $(\gamma) z^{-1}$, puisque $(\gamma) z^{-1}<\gamma$. Cela donne une contradiction.

Pour tout $z \in Z, f$ est croissante $\operatorname{sur} \operatorname{Supp}(z)$ puisqu'elle y est continue. Alors pour tout $z \in Z$,

$$
\lim _{\alpha \rightarrow(\sup \operatorname{Supp}(z))^{-}}(\alpha) f=\sup \operatorname{Supp}(z) .
$$

Par continuité à droite, $(\inf \operatorname{Supp}(z)) f=\inf \operatorname{Supp}(z)$ pour tout $z \in Z$.

Soient $S=\bigcup_{z \in Z} \operatorname{Supp}(z)$ et $L=\{\inf \operatorname{Supp}(z) \mid z \in Z\}$. Alors $S$ est ouvert et dense dans $\left[0 ; r\left[,\left.f\right|_{S}\right.\right.$ est continue, et $\left.f\right|_{L}=\operatorname{id}_{L}$.

Considérons $\alpha \in[0 ; r[\backslash S$ arbitraire. Il est facile de voir que pour tout $\beta \in] \alpha ; r[, L \cap[\alpha ; \beta[$ n'est pas vide. Donc $(\alpha) f=\alpha$ par continuité à droite en $\alpha$. Nous avons démontré que $\left.f\right|_{[0 ; r[\backslash S}=\operatorname{id}_{[0 ; r[\backslash S}$.

L'application $f$ est croissante. En effet, si $f$ est une application d'un ensemble linéairement ordonné vers lui-même, et cet ensemble est recouvert par des intervalles tels que $f$ envoie chacun d'entre eux vers lui-même d'une façon strictement croissante, alors $f$ est strictement croissante. Dans notre cas, nous avons :

$$
\left[0 ; r\left[=\bigcup_{z \in Z} \operatorname{Supp}(z) \cup \bigcup_{\alpha \in[0 ; r[\backslash S}[\alpha ; \alpha] .\right.\right.
$$

Alors $f$ est continue parce qu'elle est une surjection croissante d'un sousensemble de $\mathbb{R}$ sur un intervalle de $\mathbb{R}$.

Ces deux derniers lemmes déjà suffisent pour démontrer, à partir du résultat de Bardakov et Tolstykh, que le sous-groupe définissable de $F$ isomorphe à $\mathbb{Z} \imath \mathbb{Z}$ utilisé dans [2] est définissable dans $T$ et $V$ aussi. 
Proposition 3.3. Soient a et $b$ les éléments de $F$ montrés sur la figure 1. Alors

$$
\langle a, b\rangle=\langle b\rangle\langle\langle a\rangle \cong \mathbb{Z} \imath \mathbb{Z},
$$

et le sous-groupe $\langle a, b\rangle$ est définissable dans $F$, dans $T$, et dans $V$ par la même formule du premier ordre avec paramètres.

Démonstration. Nous considérons les mêmes éléments $x_{0}, x_{1}, a=x_{0}^{2}$, et $b=x_{1} x_{0}^{-1} x_{1}^{-1} x_{0}$ de $F$ que dans [2], voir la figure 1. Il est démontré dans [2] que :

(1) $\langle a, b\rangle=\langle b\rangle \prec\langle a\rangle \cong \mathbb{Z} \imath \mathbb{Z}$,

(2) $C_{F}\left(x_{0}\right)=\left\langle x_{0}\right\rangle$,

(3) $C_{F}\left(\left\{a^{-k} b a^{k} \mid k \in \mathbb{Z}\right\}\right)=\left\langle a^{-k} b a^{k} \mid k \in \mathbb{Z}\right\rangle$.

En particulier, $\langle a, b\rangle$ est le produit semi-direct de $C_{F}\left(\left\{a^{-k} b a^{k} \mid k \in \mathbb{Z}\right\}\right)$ et de $\langle a\rangle$, où $\langle a\rangle=\left\{x^{2} \mid x \in C_{F}\left(x_{0}\right)\right\}$.

Notons $\alpha_{k}=2^{-1+2 k}$ pour $k=-1,-2,-3, \ldots$, et $\alpha_{k}=1-2^{-1-2 k}$ pour $k=0,1,2, \ldots$. Alors

$$
0<\cdots<\alpha_{-2}<\alpha_{-1}<\alpha_{0}<\alpha_{1}<\alpha_{2}<\cdots<1
$$

Un calcul direct facilité par le lemme 2.2 montre que :

(1) $\left.\operatorname{Supp}\left(x_{0}\right)=\right] 0 ; 1[$;

(2) $(\alpha) x_{0} \geq \alpha$ pour tout $\alpha \in[0 ; 1[$;

(3) $\left.\operatorname{Supp}\left(a^{-k} b a^{k}\right)=\right] \alpha_{k} ; \alpha_{k+1}[$ pour tout $k \in \mathbb{Z}$;

(4) $(\alpha) a^{-k} b a^{k} \geq \alpha$ pour tout $\alpha \in[0 ; 1[$ et tout $k \in \mathbb{Z}$.

Si on prend $x_{0}$ comme $z$ dans le lemme 3.1, ce lemme permet de conclure que tout élément de $C_{V}\left(x_{0}\right)$ est continu. Si on pose $Z=\left\{a^{-k} b a^{k} \mid k \in \mathbb{Z}\right\}$ dans le lemme 3.2, ce lemme montre que tout élément de $C_{V}\left(\left\{a^{-k} b a^{k} \mid k \in\right.\right.$ $\mathbb{Z}\}$ ) est continu. Comme un élément de $V$ appartient à $F$ si et seulement si il est continu, on conclut que le centralisateur de l'élément $x_{0}$ et le centralisateur de l'ensemble $\left\{a^{-k} b a^{k} \mid k \in \mathbb{Z}\right\}$ sont conservés en passant de $F$ à $V$. Donc $\langle a, b\rangle$ est définissable dans $F, T$, et $V$ par la même formule du premier ordre avec paramètres.

\section{Copies DÉfinissables DE $\mathbb{Z} \imath \mathbb{Z}$}

Dans cette section, nous démontrons que tous les groupes $\mathcal{F}, \mathcal{T}$, et $\mathcal{V}$ ont de sous-groupes définissables isomorphes à $\mathbb{Z} \imath \mathbb{Z}$.

Lemme 4.1. Soient $\alpha, \beta \in[0 ; r] \cap A$ tels que $\alpha<\beta$, et soit $I=] \alpha ; \beta[$. Soit $x \in \mathcal{F}_{I}$ tel que $\operatorname{Fix}(x) \cap I \cap A=\varnothing$. Soit $\phi: \mathcal{F}_{I} \rightarrow \Lambda$ l'application $y \mapsto(\alpha) y^{\prime+}$. Soit $C$ le centralisateur de $x$ dans $\mathcal{F}_{I}$. Alors $\phi$ est un homomorphisme, et sa restriction à $C$ est injective. De même pour $\phi: \mathcal{F}_{I} \rightarrow \Lambda, y \mapsto(\beta) y^{\prime-}$.

Démonstration. Considérons seulement le cas de $\phi: \mathcal{F}_{I} \rightarrow \Lambda, y \mapsto(\alpha) y^{\prime+}$, parce que le cas de $y \mapsto(\beta) y^{\prime-}$ est analogue. Il est facile de vérifier que $\phi$ est un homomorphisme (voir le lemme 2.6). Supposons que $\phi$ ne soit pas injectif sur $C$.

Soit $y \in C$ tel que $\phi(y)=1$ mais $y \neq$ id. Soit $\gamma \in] \alpha ; \beta[$ tel que

$$
\left.y\right|_{[0 ; \gamma]}=\operatorname{id}_{[0 ; \gamma]} \quad \text { mais }(\gamma) y^{\prime+} \neq 1 .
$$


Alors $\gamma \in A$, et donc $(\gamma) x \neq \gamma$. Sans perte de généralité, supposons que $(\gamma) x>\gamma$, car sinon, alors $(\gamma) x^{-1}>\gamma$, et on peut utiliser $x^{-1}$ au lieu de $x$. Alors

$$
[0 ;(\gamma) x]=[0 ; \gamma]^{x} \subset \operatorname{Fix}(y),
$$

voir le lemme 2.3 , et donc $(\gamma) y^{\prime+}=1$. Cela donne une contradiction.

Lemme 4.2. Le centralisateur $C$ dans le lemme 4.1 est cyclique.

Ce lemme résulte de la description des centralisateurs dans $\mathcal{F}\left(r, \mathbb{R}_{+}^{*}, \mathbb{R}\right)$ obtenu par Matthew Brin et Craig Squier [6], mais pour confort du lecteur nous préférons de fournir une preuve autonome.

Démonstration du lemme $\underline{4.2}$. Notons tout d'abord que si $\Lambda$ est cyclique luimême, la conclusion de ce lemme est un corollaire évident du lemme 4.1.

Soient $\alpha, \beta, I, x, \phi$, et $C$ tels comme dans le lemme 4.1. Sans perte de généralité, supposons que $(\alpha) x^{+}>1$.

Nous allons utiliser le fait que un sous-groupe multiplicatif de $\mathbb{R}_{+}^{*}$ est soit cyclique (le sous-groupe trivial y compris), soit dense dans $\mathbb{R}_{+}^{*}$ par rapport à la topologie habituelle.

Soit $\Gamma$ l'image du groupe $C$ sous l'homomorphisme $\phi: \mathcal{F}_{I} \rightarrow \Lambda$. D'après le lemme 4.1, $\phi$ est injectif, et donc $C \cong \Gamma$. Il nous reste à montrer que $\Gamma$ n'est pas dense dans $\mathbb{R}_{+}^{*}$.

Notons que $\operatorname{Fix}(x) \cap I$ est un ensemble fini, et que

$$
\operatorname{Fix}(y) \cap I=\operatorname{Fix}(x) \cap I \text { pour tout } y \in C \backslash\{\operatorname{id}\} .
$$

En effet, il est clair que $\operatorname{Fix}(x) \cap I$ est fini parce que $\operatorname{Fix}(x) \cap I \cap A$ est vide et $A$ est dense dans $\mathbb{R}$. Considérons maintenant un $y \in C \backslash\{$ id $\}$. Par l'injectivité de $\phi,(\alpha) y^{\prime+} \neq 1$. Si $\operatorname{Fix}(y) \cap I \cap A$ était non vide, alors il aurait le plus petit élément $\gamma$, et ce $\gamma$ serait fixé par $x:(\gamma) x=x$. Cela serait une contradiction avec $\operatorname{Fix}(x) \cap I \cap A=\varnothing, \operatorname{donc} \operatorname{Fix}(y) \cap I \cap A=\varnothing$ et $\operatorname{Fix}(y) \cap I$ est fini. Comme $x$ et $y$ commutent et que chacune permute $I$, on voit que $x$ permute $\operatorname{Fix}(y) \cap I$, et que $y$ permute $\operatorname{Fix}(x) \cap I$. Comme ces ensembles sont finis, et que $x$ et $y$ préservent l'ordre, on conclut que $\operatorname{Fix}(y) \cap I \subset \operatorname{Fix}(x)$, $\operatorname{Fix}(x) \cap I \subset \operatorname{Fix}(y)$, et donc $\operatorname{Fix}(y) \cap I=\operatorname{Fix}(x) \cap I$.

Notons

$$
\beta_{0}=\min ((\operatorname{Fix}(x) \cap I) \cup\{\beta\}) ;
$$

il existe mais il n'appartient pas à $A$ sauf si $\beta_{0}=\beta$. Alors $(\gamma) x>\gamma$ pour tout $\gamma \in] \alpha ; \beta_{0}\left[\right.$, puisque $(\alpha) x^{++}>1$.

Choisissons $\left.\alpha_{1}, \beta_{1} \in\right] \alpha ; \beta_{0}\left[\right.$ tels que $x^{-1}$ soit affine sur $\left[\alpha ; \alpha_{1}\right]$ et $x$ soit affine sur $\left[\beta_{1} ; \beta_{0}\left[\right.\right.$. Alors $x$ est aussi affine sur $\left[\alpha ;\left(\alpha_{1}\right) x^{-1}\right]$, et $x^{-1}$ est affine $\operatorname{sur}\left[\left(\beta_{1}\right) x ; \beta_{0}[\right.$.

Démontrons maintenant que si $y \in C$ et $(\alpha) y^{\prime+}>1$, alors $y$ est affine sur $\left[\alpha ;\left(\alpha_{1}\right) y^{-1}\right]$ et sur $\left[\beta_{1} ; \beta_{0}[\right.$. Considérons un tel $y$. Donc $(\gamma) y>\gamma$ pour tout $\gamma \in] \alpha ; \beta_{0}[$. Alors pour tout $\left.\gamma \in] \alpha ; \alpha_{1}\right]$,

$$
\left.\left.\left.x^{-1}\right|_{\left[\alpha ; \alpha_{1}\right]} \cdot y^{-1}\right|_{\left[\alpha ;(\gamma) x^{-1}\right]} \cdot x\right|_{\left[\alpha ;\left(\alpha_{1}\right) x^{-1}\right]}=\left.y^{-1}\right|_{[\alpha ; \gamma]},
$$

et pour tout $\gamma \in\left[\beta_{1} ; \beta_{0}[\right.$,

$$
\left.\left.\left.x\right|_{\left[\beta_{1} ; \beta_{0}[\right.} \cdot y\right|_{\left[(\gamma) x ; \beta_{0}[\right.} \cdot x^{-1}\right|_{\left[\left(\beta_{1}\right) x ; \beta_{0}[\right.}=\left.y\right|_{\left[\gamma ; \beta_{0}[\right.} .
$$

Ces égalités évidentes impliquent que : 
(1) pour tout $\left.\gamma \in] \alpha ; \alpha_{1}\right], y^{-1}$ est affine sur $[\alpha ; \gamma]$ dès qu'elle est affine sur $\left[\alpha ;(\gamma) x^{-1}\right]$

(2) pour tout $\gamma \in\left[\beta_{1} ; \beta_{0}\left[\right.\right.$, y est affine sur $\left[\gamma ; \beta_{0}[\right.$ dès qu'elle l'est sur $\left[(\gamma) x ; \beta_{0}[\right.$.

Ceci n'est possible que si $y^{-1}$ est affine sur $\left[\alpha ; \alpha_{1}\right]$, et $y$ est affine sur $\left[\beta_{1} ; \beta_{0}[\right.$.

Clairement $\lim _{n \rightarrow+\infty}(\gamma) x^{n}=\beta_{0}$ pour tout $\left.\gamma \in\right] \alpha ; \beta_{0}[$ (voir la preuve du lemme 2.5). Choisissons un entier $n$ tel que

$$
\left(\alpha_{1}\right) x^{n}>\beta_{1}, \quad \text { et donc }\left(\beta_{1}\right) x^{-n}<\alpha_{1} .
$$

Notons $p=\left(\beta_{1}\right)\left(x^{-n}\right)^{\prime+}$. Alors $p^{-1}=\left(\left(\beta_{1}\right) x^{-n}\right)\left(x^{n}\right)^{\prime+}$. Choisissons $\gamma$ dans $\left(\left(\beta_{1}\right) x^{-n} ; \alpha_{1}\right]$ tel que $x^{n}$ soit affine sur $\left[\left(\beta_{1}\right) x^{-n} ; \gamma\right]$ (avec la pente $\left.p^{-1}\right)$.

Supposons que $y$ soit un élément de $C$ tel que

$$
1<(y) \phi<\frac{\gamma-\alpha}{\left(\beta_{1}\right) x^{-n}-\alpha} .
$$

Alors $y^{-1}$ est affine sur $\left[\alpha ; \alpha_{1}\right]$, $y$ est affine sur $\left[\beta_{1} ; \beta_{0}\right.$ [, et $\left(\beta_{0}\right) y^{-}<1$ (parce que $(\gamma) y>\gamma$ pour tout $\gamma \in] \alpha ; \beta_{0}[)$. Notons $q=(\alpha) y^{\prime+}=(y) \phi$. Alors

$$
\left(\beta_{1}\right) x^{-n}<\alpha+(\gamma-\alpha) q^{-1}=(\gamma) y^{-1} \leq \alpha+\left(\alpha_{1}-\alpha\right) q^{-1}=\left(\alpha_{1}\right) y^{-1},
$$

et par conséquent $\left(\beta_{1}\right) x^{-n}<\left(\beta_{1}\right) x^{-n} y<\gamma$. Comme $y=x^{-n} y x^{n}$, on calcule

$$
\begin{aligned}
\left(\beta_{1}\right) y^{\prime+} & =\left(\beta_{1}\right)\left(x^{-n}\right)^{\prime+} \cdot\left(\left(\beta_{1}\right) x^{-n}\right) y^{\prime+} \cdot\left(\left(\beta_{1}\right) x^{-n} y\right)\left(x^{n}\right)^{\prime+} \\
& =p q p^{-1}=q>1 .
\end{aligned}
$$

Comme $\left(\beta_{1}\right) y^{\prime+}=\left(\beta_{0}\right) y^{\prime-}<1$, cela donne une contradiction, ce qui signifie que

$$
\Gamma \cap] 1 ; \frac{\gamma-\alpha}{\left(\beta_{1}\right) x^{-n}-\alpha}[=\varnothing .
$$

Lemme 4.3. Soient $\alpha, \beta \in[0 ; r] \cap A$ tels que $\alpha<\beta$. Soient $p, q \in \Lambda$ tels que $p>1>q$. Alors il existe $x \in \mathcal{F}^{\uparrow}$ tel que $\left.\operatorname{Supp}(x)=\right] \alpha ; \beta\left[,(\alpha) x^{++}=p\right.$, et que $(\beta) x^{--}=q$.

Démonstration. Soit $s$ un élément de $\Lambda$ tel que $(2+p+q) s \leq 1$. Notons $l=$ $\beta-\alpha$. Faisons deux partitions de l'intervalle $[\alpha ; \beta]$ : la première - en sousintervalles de longueurs $s l, q s l,(1-(2+p+q) s) l, p s l$, et $s l$, dans cet ordre, et la deuxième - en sous-intervalles de longueurs $p s l, s l,(1-(2+p+q) s) l$, $s l$, et $q s l$, dans cet ordre. Soit $x$ l'application continue $[0 ; r] \rightarrow[0 ; r]$ qui est l'identité sur $[0 ; \alpha] \cup[\beta ; r]$, et qui envoie chaque intervalle de la première partition de $[\alpha ; \beta]$ de la façon affine sur l'intervalle correspondante de la deuxième. Il est facile de vérifier que $\operatorname{Supp}(x)=] \alpha ; \beta\left[,(\alpha) x^{++}=p,(\beta) x^{--}=\right.$ $q$, et que $\left.x\right|_{[0 ; r[} \in \mathcal{F}^{\uparrow}$.

Choisissons $a \in \mathcal{F}^{\uparrow}$ tel que $\left.\operatorname{Supp}(a)=\right] 0 ; r\left[\right.$. Choisissons $\left.\alpha_{0} \in\right] 0 ; r[\cap A$ arbitrairement. Pour tout $k \in \mathbb{Z}$, définissons $\alpha_{k}=\left(\alpha_{0}\right) a^{k}$. Notons que

$$
\begin{gathered}
0<\cdots<\alpha_{-2}<\alpha_{-1}<\alpha_{0}<\alpha_{1}<\alpha_{2}<\cdots<r, \\
\text { et que } \quad \lim _{n \rightarrow-\infty} \alpha_{n}=0, \quad \lim _{n \rightarrow+\infty} \alpha_{n}=r
\end{gathered}
$$

(voir le lemme 2.5 et la remarque 2.7). Choisissons $b \in \mathcal{F}^{\uparrow}$ tel que $\operatorname{Supp}(b)=$ ]$\alpha_{0} ; \alpha_{1}[$ (voir la figure 2). Grâce au lemme 4.3, $a$ et $b$ existent. 




FIG. 2. Un exemple des applications $a$ et $b$.

Lemme 4.4. Le groupe engendré par a et b est isomorphe au produit en couronne restreint $\mathbb{Z} \imath \mathbb{Z} ;$ plus précisément,

$$
\langle a, b\rangle=\langle b\rangle ح\langle a\rangle, \quad\langle b\rangle \cong\langle a\rangle \cong \mathbb{Z} .
$$

Démonstration. Notons tout d'abord que $\left.\operatorname{Supp}\left(a^{-k} b a^{k}\right)=\right] \alpha_{k} ; \alpha_{k+1}[\operatorname{pour}$ tout $k \in \mathbb{Z}$ (voir le lemme 2.2). En particulier, les supports des applications $a^{-k} b a^{k}, k \in \mathbb{Z}$, sont deux-à-deux disjoints, et $\left.\operatorname{Supp}(a)=\right] 0 ; r[$ n'est égal au support d'aucun élément du groupe $\left\langle a^{-k} b a^{k} \mid k \in \mathbb{Z}\right\rangle$. Donc

$$
\left\langle a^{-k} b a^{k} \mid k \in \mathbb{Z}\right\rangle=\bigoplus_{k \in \mathbb{Z}}\left\langle a^{-k} b a^{k}\right\rangle \quad \text { et } \quad\left\langle a^{-k} b a^{k} \mid k \in \mathbb{Z}\right\rangle \cap\langle a\rangle=\{\mathrm{id}\},
$$

d'où $\langle a, b\rangle=\left\langle a^{-k} b a^{k} \mid k \in \mathbb{Z}\right\rangle \rtimes\langle a\rangle=\langle b\rangle ح\langle a\rangle$. Comme $\mathcal{F}$ est sans torsion, $\langle a\rangle \cong\langle b\rangle \cong \mathbb{Z}$.

Dans le reste de cette section, $G$ est un sous-groupe de $\mathcal{V}\left(r, \mathbb{R}_{+}^{*}, \mathbb{R}\right)$ tel que

$$
G \cap \mathcal{F}\left(r, \mathbb{R}_{+}^{*}, \mathbb{R}\right)=\mathcal{F}=\mathcal{F}(r, \Lambda, A)
$$

(il est même possible de généraliser certains résultats de cette section au cas où $G$ satisferait une condition plus faible que celle-ci).

Proposition 4.5. Soient $a, b$, et $G$ les éléments et le groupe définis cidessus. Alors il existe une formule $\phi$ du premier ordre avec a et $b$ comme paramètres et avec une seule variable libre tel que $\langle a, b\rangle$ soit défini dans $G$ par $\phi$. De plus, on peut choisir $\phi$ en fonction seulement de $a, b$, et $\mathcal{F}$ (sans avoir connaître $G$ tout entier).

Afin de trouver une telle $\phi$ et ainsi prouver cette proposition, choisissons tout d'abord $c, d \in \mathcal{F}$ et $s, t \in \mathbb{N}$ tels que 
(1) c soit un générateur du centralisateur de $a$ dans $\mathcal{F}$,

(2) $d$ soit un générateur du centralisateur de $b$ dans $\mathcal{F}_{] \alpha_{0} ; \alpha_{1}[}$,

(3) $a=c^{s}$ et $b=d^{t}$.

Les éléments $c$ et $d$ et les nombres $s$ et $t$ existent d'après le lemme 4.2. D'après le lemme 2.4, $\operatorname{Supp}(c)=] 0 ; r[$ et $\operatorname{Supp}(d)=] \alpha_{0} ; \alpha_{1}\left[\right.$. Clairement $\overline{c, d} \in \mathcal{F}^{\uparrow}$.

Notons que $a^{-k} d a^{k} \in \mathcal{F}^{\uparrow}$ et que $\left.\operatorname{Supp}\left(a^{-k} d a^{k}\right)=\right] \alpha_{k} ; \alpha_{k+1}$ [ pour tout $k$. Comme pour tout $k$ la conjugaison par $a^{k}$ est un automorphisme de $\mathcal{F}$ qui envoie $\mathcal{F}_{] \alpha_{0} ; \alpha_{1}[}$ sur $\mathcal{F}_{\alpha_{k} ; \alpha_{k+1}[}, a^{-k} d a^{k}$ est un générateur du centralisateur de

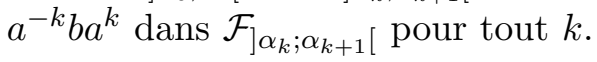

Lemme 4.6. Le centralisateur de $\left\{a^{-k} b a^{k} \mid k \in \mathbb{Z}\right\}$ dans $\mathcal{F}$ est engendré $\operatorname{par}\left\{a^{-k} d a^{k} \mid k \in \mathbb{Z}\right\}$.

Démonstration. L'inclusion

$$
C_{\mathcal{F}}\left(\left\{a^{-k} b a^{k} \mid k \in \mathbb{Z}\right\}\right) \supset\left\langle a^{-k} d a^{k} \mid k \in \mathbb{Z}\right\rangle
$$

est évidente, il reste à montrer l'inclusion inverse. Alors soit $x$ un élément arbitraire de $\mathcal{F}$ qui commute avec tout $a^{-k} b a^{k}, k \in \mathbb{Z}$.

D'après le lemme $2.2, x$ permute chacun des intervalles $] \alpha_{k} ; \alpha_{k+1}[, k \in \mathbb{Z}$. Par continuité et monotonicité, $\left(\alpha_{k}\right) x=\alpha_{k}$ pour tout $k$. Pour tout $k \in \mathbb{Z}$, soit $y_{k}$ la permutation de $[0 ; r[$ telle que

$$
\left.y_{k}\right|_{\left[\alpha_{k} ; \alpha_{k+1}\right]}=\left.x\right|_{\left[\alpha_{k} ; \alpha_{k+1}\right]} \quad \text { et }\left.\quad y_{k}\right|_{\left[0 ; \alpha_{k}\right] \cup\left[\alpha_{k+1} ; r[\right.}=\operatorname{id}_{\left[0 ; \alpha_{k}\right] \cup\left[\alpha_{k+1} ; r[\right.} \cdot
$$

Alors pour tout $k \in \mathbb{Z}, y_{k} \in \mathcal{F}_{\alpha_{k} ; \alpha_{k+1}[}$ et $y_{k}$ commute avec $a^{-k} b a^{k}$. Donc $y_{k} \in\left\langle a^{-k} d a^{k}\right\rangle$ pour tout $k$.

Choisissons $\beta, \gamma \in] 0 ; r$ tels que $x$ soit affine sur $[0 ; \beta]$ et sur $[\gamma ; r[$. Alors $\left.x\right|_{[0 ; \beta] \cup[\gamma ; r[}=\operatorname{id}_{[0 ; \beta] \cup[\gamma ; r[}$. Choisissons $n \in \mathbb{N}$ tel que $\left.\left.\alpha_{-n} \in\right] 0 ; \beta\right]$ et $\alpha_{n+1} \in$ $\left[\gamma ; r[\right.$. Alors $\operatorname{Supp}(x) \subset] \alpha_{-n} ; \alpha_{n+1}[$, et donc

$$
x=y_{-n} y_{-n+1} \cdots y_{n-1} y_{n} \in\left\langle a^{-k} d a^{k} \mid k \in \mathbb{Z}\right\rangle .
$$

Lemme 4.7. Le centralisateur de l'élément a et le centralisateur de l'ensemble $\left\{a^{-k} b a^{k} \mid k \in \mathbb{Z}\right\}$ sont conservés en passant de $\mathcal{F}$ à $G$.

Démonstration. Tous éléments de $C_{G}(a)$ et de $C_{G}\left(\left\{a^{-k} b a^{k} \mid k \in \mathbb{Z}\right\}\right)$ sont continus d'après les lemmes 3.1 et 3.2. Comme un élément de $G$ appartient à $\mathcal{F}$ si et seulement si il est continu, la preuve est terminée.

Le groupe $\langle c\rangle$ est définissable dans $G$ avec le paramètre $a$ puisqu'il est le centralisateur de $a$ (voir le lemme 4.7). Le groupe $\langle a\rangle$ est définissable dans $G$ avec le même paramètre parce que

$$
\langle a\rangle=\left\{x^{s} \mid x \in\langle c\rangle\right\} .
$$

Donc l'ensemble $\left\{a^{-k} b a^{k} \mid k \in \mathbb{Z}\right\}$ est définissable dans $G$ avec les paramètres $a$ et $b$, et ainsi son centralisateur l'est. Par les lemmes 4.6 et 4.7, le centralisateur de l'ensemble $\left\{a^{-k} b a^{k} \mid k \in \mathbb{Z}\right\}$ dans $G$ est le groupe $\left\langle a^{-k} d a^{k} \mid k \in \mathbb{Z}\right\rangle$. Comme

$$
\left\langle a^{-k} b a^{k} \mid k \in \mathbb{Z}\right\rangle=\left\{x^{t} \mid x \in\left\langle a^{-k} d a^{k} \mid k \in \mathbb{Z}\right\rangle\right\},
$$


le groupe $\left\langle a^{-k} b a^{k} \mid k \in \mathbb{Z}\right\rangle$ est définissable avec les mêmes paramètres. Le groupe $\langle a, b\rangle$ est définissable avec les paramètres $a$ et $b$ puisqu'il est le produit semi-direct de $\langle a\rangle$ et $\left\langle a^{-k} b a^{k} \mid k \in \mathbb{Z}\right\rangle$.

La formule suivante définit $\langle a, b\rangle$ dans $G$ et ne dépend que de $a, b, s$, et $t$ :

$$
\begin{aligned}
\phi(x)=\left\ulcorner( \exists y , z ) \left( x=y^{s} z^{t}\right.\right. & \wedge y a=a y \\
& \left.\left.\wedge(\forall w)\left(w a=a w \rightarrow z w^{-s} b w^{s}=w^{-s} b w^{s} z\right)\right)\right\urcorner .
\end{aligned}
$$

Nous avons démontré la proposition 4.5. Nous en déduisons le corollaire suivant.

Corollaire 4.8. Le groupe $\mathcal{F}$ a des sous-groupes isomorphes $\grave{a} \mathbb{Z} \backslash \mathbb{Z}$ et définissables avec paramètres dans $\mathcal{F}$, dans $\mathcal{T}$, et dans $\mathcal{V}$.

\section{INTERPRÉTATIONS DE L'ARITHMÉtiQUe}

Dans cette section, nous complétons notre démonstration de l'interprétabilité de l'Arithmétique dans $\mathcal{F}, \mathcal{T}$, et $\mathcal{V}$ avec paramètres. En outre, au cas du groupe $F$, ou plus généralement du groupe $\mathcal{F}$ avec $\Lambda$ cyclique non trivial, nous présentons une interprétation de l'Arithmétique qui ne nécessite pas de paramètres.

Apparemment il est bien connu aux spécialistes que chaque groupe virtuellement résoluble de type fini qui n'est pas virtuellement abélien interprète l'Arithmétique (voir [22, 23], et aussi [10]). Pour confort du lecteur, nous presentons ici notre preuve autonome pour le groupe $\mathbb{Z} \imath \mathbb{Z}$.

Lemme 5.1. Le groupe $\mathbb{Z} \imath \mathbb{Z}$ interprète l'Arithmétique avec paramètres. Plus précisément, soit $\mathbb{Z} \prec \mathbb{Z}=\langle b\rangle\langle\langle a\rangle,\langle b\rangle \cong\langle a\rangle \cong \mathbb{Z}$, alors la bijection

$$
f:\left\{a^{n} \mid n \in \mathbb{N}\right\} \rightarrow \mathbb{N}, \quad a^{n} \mapsto n
$$

est une interprétation de $(\mathbb{N},+, \times)$ dans $(\mathbb{Z} \imath \mathbb{Z}, \times)$ avec paramètres.

Démonstration. Notons

$$
G=\mathbb{Z} \imath \mathbb{Z}=\langle b\rangle \imath\langle a\rangle \quad \text { et } \quad H=\left\langle a^{-k} b a^{k} \mid k \in \mathbb{Z}\right\rangle .
$$

Rappelons que $G=H \rtimes\langle a\rangle$, et que $H$ est un groupe abélien libre avec la base $\left(a^{-k} b a^{k}\right)_{k \in \mathbb{Z}}$. On peut vérifier facilement que $\langle a\rangle=C_{G}(a)$, et que $H=C_{G}(b)$.

Considérons la bijection

$$
g:\langle a\rangle \rightarrow \mathbb{Z}, \quad a^{n} \mapsto n .
$$

Il suffira de démontrer que $g$ est une interprétation de $(\mathbb{Z},+, \times)$ dans $(G, \times)$. En effet, $(\mathbb{N},+, \times)$ est une sous-structure de $(\mathbb{Z},+, \times)$, et $\mathbb{N}$ est 0 -définissable dans $(\mathbb{Z},+, \times)$ parce que, d'après le théorème des quatre carrés de Lagrange, tout entier positif est la somme de quatre carrés.

L'ensemble de départ de $g$ est le centralisateur de $a$, donc définissable. L'opération induite sur $\langle a\rangle$ par l'addition de $\mathbb{Z}$ via $g$ est tout simplement la restriction de la multiplication de $G$, donc 0 -définissable. Il reste à démontrer que l'opération induite sur $\langle a\rangle$ par la multiplication de $\mathbb{Z}$ via $g$ est définissable.

Remarquons les faits suivants : 
(1) pour tout $x \in G \backslash H, C_{G}(x)$ est cyclique,

(2) pour tout $n \in \mathbb{Z} \backslash\{0\}, C_{G}\left(b a^{n}\right)=\left\langle b a^{n}\right\rangle$,

(3) pour tout $n \in \mathbb{Z}, H C_{G}\left(b a^{n}\right)=H\left\langle a^{n}\right\rangle$.

(Le deuxième fait est dû à l'homomorphisme $G \rightarrow \mathbb{Z}, a \mapsto 0, b \mapsto 1$.)

Notons | la relation de la divisibilité dans $\mathbb{Z}$. Observons que pour tous $m, n \in \mathbb{Z}$,

$$
m \mid n \Leftrightarrow H C_{G}\left(b a^{m}\right) \supset H C_{G}\left(b a^{n}\right) .
$$

La relation $H C_{G}(b x) \supset H C_{G}(b y)$ entre $x, y \in G$ s'exprime par une formule du premier ordre avec le paramètre $b$. Donc la relation induite sur $\langle a\rangle$ par $\mid$ via $g$ est définissable.

La multiplication dans $\mathbb{Z}$ est définissable à partir de l'addition, la divisibilité, et la constante 1 grâce aux équivalences suivantes satisfaites dans $\mathbb{Z}$ :

$$
\begin{aligned}
n=k(k+1) & \leftrightarrow(\forall m)(n|m \leftrightarrow k| m \wedge(k+1) \mid m) \wedge(2 k+1) \mid(2 n-k), \\
n=k l & \leftrightarrow(k+l)(k+l+1)=k(k+1)+l(l+1)+2 n
\end{aligned}
$$

(voir $[29, \S 5 \mathrm{a}]$ pour les détails). Donc l'opération induite sur $\langle a\rangle$ par la multiplication de $\mathbb{Z}$ via $g$ est définissable.

Le théorème A (voir l'introduction) est un corollaire de la proposition 4.5 et du lemme 5.1. Afin de démontrer le théorème $\mathrm{B}$, nous construirons des 0 -interprétations nouvelles de l'Arithmétiques dans des groupes de genre $\mathcal{F}$.

Proposition 5.2. Si $\Lambda$ est cyclique, $\Lambda=\langle p\rangle$, alors l'application

$$
f:\left\{x \in \mathcal{F} \mid(0) x^{\prime+}=(r) x^{\prime-}>1\right\} \rightarrow \mathbb{N}, \quad x \mapsto \log _{p}\left((0) x^{\prime+}\right)
$$

est une interprétation de $(\mathbb{N},+, \times)$ dans $(\mathcal{F}, \times)$ sans paramètres.

Une des idées principales de la preuve de la proposition 5.2 est l'usage des centralisateurs de paires d'éléments. ${ }^{5}$ Le lemme suivant est similaire au théorème 5.5 dans $[6]$.

Lemme 5.3. Soit $H$ un sous-groupe de $\mathcal{F}$. Alors $H$ est le centralisateur d'un élément si et seulement si $H$ se décompose en un produit direct des sous-groupes $H_{1}, \ldots, H_{n}, n \in \mathbb{N}$, tels qu'il existe $\alpha_{0}, \ldots, \alpha_{n} \in A$ tels que :

(1) $0=\alpha_{0}<\alpha_{1}<\cdots<\alpha_{n}=r$;

(2) pour tout $i=1, \ldots, n$, soit $H_{i}=\mathcal{F}_{] \alpha_{i-1} ; \alpha_{i}}$, soit il existe $x$ dans $\mathcal{F}_{] \alpha_{i-1} ; \alpha_{i}[}$ tel que $H_{i}$ soit le centralisateur de $x$ dans $\mathcal{F}_{] \alpha_{i-1} ; \alpha_{i}}$, et que $H_{i}=\langle x\rangle$

(3) pour tout $i=1, \ldots, n-1$, si $H_{i}=\mathcal{F}_{] \alpha_{i-1} ; \alpha_{i}}$, alors $H_{i+1} \neq \mathcal{F}_{] \alpha_{i} ; \alpha_{i+1}}[$.

Démonstration. Soient $x \in \mathcal{F}$ et $H=C_{\mathcal{F}}(x)$. Choisissons $\alpha_{0}, \ldots, \alpha_{n}$ tels que $0=\alpha_{0}<\alpha_{1}<\cdots<\alpha_{n}=r$ et que

$$
\left\{\alpha_{1}, \ldots, \alpha_{n-1}\right\}=\{\alpha \in] 0 ; r\left[\cap A \cap \operatorname{Fix}(x) \mid(\alpha) x^{\prime-} \neq 1 \text { ou }(\alpha) x^{\prime+} \neq 1\right\} .
$$

Pour tout $i=1, \ldots, n$, soit $x_{i} \in \mathcal{F}_{] \alpha_{i-1} ; \alpha_{i}[}$ tel que $\left.x_{i}\right|_{] \alpha_{i-1} ; \alpha_{i}[}=\left.x\right|_{] \alpha_{i-1} ; \alpha_{i}}$, et soit $H_{i}$ le centralisateur de $x_{i}$ dans $\mathcal{F}_{] \alpha_{i-1} ; \alpha_{i}}$. Notons que $x=x_{1} \cdots x_{n}$. Pour tout $i=1, \ldots, n$, si $x_{i} \neq$ id, alors $H_{i}$ est cyclique (voir le lemme 4.2).

\footnotetext{
${ }^{5}$ Les centralisateurs dans $\mathcal{F}\left(r, \mathbb{R}_{+}^{*}, \mathbb{R}\right)$ ont été décrits par Brin et Squier [6]. Collin Bleak et autres [5] ont récemment annoncé une classification de tous les centralisateurs dans $\mathcal{T}\left(1,\langle n\rangle, \mathbb{Z}\left[\frac{1}{n}\right]\right)$ et $\mathcal{V}\left(1,\langle n\rangle, \mathbb{Z}\left[\frac{1}{n}\right]\right), n=2,3, \ldots$
} 
Pareillement au lemme 2.3, il est facile à prouver que tout élément $y$ de $H$ permute l'ensemble $\left\{\alpha_{0}, \ldots, \alpha_{n-1}\right\}$, et donc, comme cet ensemble est fini, $y$ fixe tous ses éléments. Donc $H=H_{1} \times \cdots \times H_{n}$.

Réciproquement, supposons que $H=H_{1} \times \cdots \times H_{n}, n \in \mathbb{N}$, et que les sous-groupes $H_{1}, \ldots, H_{n}$ et les points $\alpha_{0}, \ldots, \alpha_{n} \in A$ soient comme dans l'énoncé de ce lemme. Pour tout $i=1, \ldots, n$, soit $x_{i} \in \mathcal{F}_{] \alpha_{i-1} ; \alpha_{i} \text { [ }}$ tel que $H_{i}$ soit le centralisateur de $x_{i}$ dans $\mathcal{F}_{] \alpha_{i-1} ; \alpha_{i}[}$. Alors $H=C_{\mathcal{F}}\left(x_{1} \cdots x_{n}\right)$.

Lemme 5.4. Soit $H$ un sous-groupe de $\mathcal{F}$. Alors $H$ est le centralisateur d'une paire d'éléments (éventuellement identiques) si et seulement si $H$ se décompose en un produit direct des sous-groupes $H_{1}, \ldots, H_{n}, n \in \mathbb{N}$, tels qu'il existe $\alpha_{0}, \ldots, \alpha_{n} \in A$ tels que :

(1) $0=\alpha_{0}<\alpha_{1}<\cdots<\alpha_{n}=r$;

(2) pour tout $i=1, \ldots, n$, soit $H_{i}=\{$ id $\}$, soit $H_{i}=\mathcal{F}_{] \alpha_{i-1} ; \alpha_{i}}$, soit il existe $x$ dans $\mathcal{F}_{] \alpha_{i-1} ; \alpha_{i}}$ tel que $H_{i}$ soit le centralisateur de $x$ dans $\mathcal{F}_{] \alpha_{i-1} ; \alpha_{i}}$, et que $H_{i}=\langle x\rangle$;

(3) pour tout $i=1, \ldots, n-1$, si $H_{i}=\mathcal{F}_{] \alpha_{i-1} ; \alpha_{i}}$, alors $H_{i+1} \neq \mathcal{F}_{] \alpha_{i} ; \alpha_{i+1}}$.

Démonstration. Ce lemme est un corollaire facile du lemme 5.3 et du fait que pour tous $\alpha, \beta \in A$ tels que $0<\alpha<\beta<r$, il existe $x, y \in \mathcal{F}$ tels que $\operatorname{Supp}(x)=\operatorname{Supp}(y)=] \alpha ; \beta[$ et que $x y \neq y x$, et donc le centralisateur de $\{x, y\}$ dans $\mathcal{F}_{] \alpha ; \beta[}$ est trivial (voir les lemmes 4.2 et 4.3 ).

Démonstration de la proposition 5.2. Sans perte de généralité, supposons que $p>1$.

Notons $\mathcal{F}^{\circ}$ le sous-groupe de $\mathcal{F}$ formé des éléments qui sont l'identité aux voisinages de 0 et de $r$ :

$$
\mathcal{F}^{\circ}=\left\{x \in \mathcal{F} \mid(0) x^{\prime+}=(r) x^{{ }^{-}}=1\right\} .
$$

Notons $B$ l'ensemble de départ de $f$ :

$$
B=\left\{x \in \mathcal{F} \mid(0) x^{\prime+}=(r) x^{\prime-}>1\right\} .
$$

Remarquons que pour deux éléments $x$ et $y$ de $B, f(x)=f(y)$ si et seulement si $x y^{-1} \in \mathcal{F}^{\circ}$ (voir le lemme 4.1).

Le lemme 4.3 permet de conclure que $f$ est une surjection sur $\mathbb{N}$ (voir la figure 3 par exemple). Il nous suffira de démontrer que l'ensemble $B$, le groupe $\mathcal{F}^{\circ}$, et les relations induites sur $B$ via $f$ par l'addition et la divisibilité de $\mathbb{N}$ sont tous 0-définissables (voir la remarque 2.10). En effet, la multiplication est 0 -définissable dans $\mathbb{N}$ à partir de l'addition et de la divisibilité — voir $[29, \S 4 \mathrm{~b}]$ ou la preuve du lemme 5.1.

Notons

$$
\begin{aligned}
& S=\left\{\mathcal{F}_{] \alpha ; \beta[} \mid \alpha, \beta \in A, 0 \leq \alpha<\beta \leq r\right\}, \\
& S_{0}=\left\{\mathcal{F}_{] \alpha ; \beta[} \mid \alpha, \beta \in A, 0<\alpha<\beta<r\right\}, \\
& S_{1}=\left\{\mathcal{F}_{] 0 ; \beta[} \mid \beta \in A, 0<\beta<r\right\} \\
& \cup\left\{\mathcal{F}_{] \alpha ; r[} \mid \alpha \in A, 0<\alpha<r\right\} .
\end{aligned}
$$

Alors non seulement la famille $S$ est uniformément définissable, mais surtout il existe deux formules du premier ordre $\phi\left(x_{1}, x_{2}, x_{3}\right)$ et $\psi\left(x_{1}, x_{2}\right)$ (dans le langage des groupes) sans paramètres telles que : 


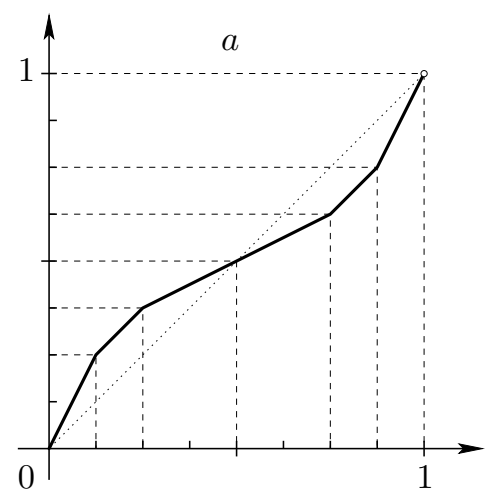

Fig. 3. Un élément $a$ de $F$ tel que $(0) a^{++}=(1) a^{--}=2$.

(1) pour tous $\alpha, \beta \in A$ tels que $0 \leq \alpha<\beta \leq r$, il existe $x, y \in \mathcal{F}$ tels que $\mathcal{F}=\psi(x, y)$ et que $\mathcal{F}_{] \alpha ; \beta[}=\{z \in \mathcal{F} \mid \mathcal{F} \models \phi(x, y, z)\}$;

(2) pour tous $x, y \in \mathcal{F}$ tels que $\mathcal{F}=\psi(x, y)$, il existe $\alpha, \beta \in A$ tels que $0 \leq \alpha<\beta \leq r$ et que $\mathcal{F}_{] \alpha ; \beta[}=\{z \in \mathcal{F} \mid \mathcal{F}=\phi(x, y, z)\}$.

En effet, une partie de $\mathcal{F}$ est de la forme $\mathcal{F}_{] \alpha ; \beta}$, où $\alpha, \beta \in A$ et $0 \leq \alpha<$ $\beta \leq r$, si et seulement si elle est le centralisateur d'une paire, qui n'est pas abélien, et qui ne se décompose pas comme le produit direct de deux autres centralisateurs de paires (voir le lemme 5.4). Tout cela s'exprime au premier ordre. Les familles $S_{0}$ et $S_{1}$ de parties de $\mathcal{F}$ sont « uniformément définissables sans paramètres » au même sens que $S$ parce que

$$
\begin{aligned}
& S_{0}=\left\{H_{1} \cap H_{2} \mid H_{1}, H_{2} \in S, H_{1} \not \subset H_{2}, \text { et } H_{2} \not \subset H_{1}\right\}, \\
& S_{1}=S \backslash\left(S_{0} \cup\{\mathcal{F}\}\right) .
\end{aligned}
$$

Comme $\mathcal{F}^{\circ}=\bigcup S_{0}$, ce sous-groupe est 0 -définissable. ${ }^{6}$

Notons

$$
\begin{aligned}
E & =\left\{x \in \mathcal{F} \mid(0) x^{\prime+} \neq 1 \text { ou }(r) x^{\prime-} \neq 1\right\}=\mathcal{F} \backslash \bigcup S_{0}=\mathcal{F} \backslash \mathcal{F}^{\circ}, \\
E_{2} & =\left\{x \in \mathcal{F} \mid(0) x^{\prime+} \neq 1 \text { et }(r) x^{\prime-} \neq 1\right\}=\mathcal{F} \backslash \bigcup S_{1}=E \backslash \bigcup S_{1} .
\end{aligned}
$$

Ces ensembles sont 0-définissables.

Notons

$$
\begin{aligned}
& P^{+}=\left\{x \in \mathcal{F} \mid(0) x^{++}>1 \text { et }(r) x^{\prime-}>1\right\}, \\
& P^{-}=\left\{x \in \mathcal{F} \mid(0) x^{\prime+}<1 \text { et }(r) x^{\prime-}<1\right\},
\end{aligned}
$$

et $P=P^{+} \cup P^{-}$. Ces ensembles sont 0 -définissables : pour tout $x \in \mathcal{F}$,

$$
x \in P^{+} \Leftrightarrow\left(\exists X \in S_{0}\right)\left(\forall Y \in S_{1}\right)\left(Y \supset X \rightarrow x^{-1} Y x \varsubsetneqq Y\right),
$$

et

$$
x \in P^{-} \Leftrightarrow\left(\exists X \in S_{0}\right)\left(\forall Y \in S_{1}\right)\left(Y \supset X \rightarrow x^{-1} Y x \supsetneqq Y\right) .
$$

\footnotetext{
${ }^{6}$ Dans le cas où $\mathcal{F}=F$, il existe une preuve plus naturelle pour montrer que $\mathcal{F}^{\circ}$ est définissable dans $\mathcal{F}$, comme dans ce cas $\mathcal{F}^{\circ}=F^{\circ}=[F, F]=[\mathcal{F}, \mathcal{F}]$ (voir [ $4.1]$ ), et que tout élément de $[\mathcal{F}, \mathcal{F}]$ est le produit de deux commutateurs (voir l'appendice). En général, $\mathcal{F}^{\circ}$ et $[\mathcal{F}, \mathcal{F}]$ ne sont pas égaux (voir $[7$, section $4 \mathrm{D}]$ ).
} 
Il a été utilisé ici que pour tous $\alpha, \beta$ et pour tout $x \in \mathcal{F}$,

$$
x^{-1} \mathcal{F}_{] \alpha ; \beta} x=\mathcal{F}_{](\alpha) x ;(\beta) x[} .
$$

Notons

$$
U=\left\{x \in \mathcal{F} \mid(0) x^{++}=\left((r) x^{\prime-}\right)^{-1} \in\left\{p^{ \pm 1}\right\}\right\} .
$$

Alors $U$ est 0-définissable : $U \subset E_{2} \backslash P$, et pour tout $x \in E_{2} \backslash P$,

$$
\begin{aligned}
x \in U \Leftrightarrow(\forall y \in P)\left(\forall z \in \mathcal{F}^{\circ}\right) & \left(\exists w_{1}, w_{2} \in C_{\mathcal{F}}(x z)\right) \\
& \left(\left(w_{1} w_{2}^{-1} \in E_{2}\right) \wedge\left(y w_{1}, y w_{2} \notin E_{2}\right)\right) .
\end{aligned}
$$

Afin de faciliter la lecture de la dernière formule, remarquons que $x y^{-1} \notin E_{2}$ signifie exactement que soit $(0) x^{\prime+}=(0) y^{\prime+}$, soit $(r) x^{\prime-}=(r) y^{\prime-}$. Alors l'implication « $\Rightarrow \gg$ est facile à démontrer : on peut toujours trouver de tels $w_{1}, w_{2}$ même dans $\langle x z\rangle$. La direction « $\Leftarrow »$ est moins évidente, démontronsla par contraposé.

Soit $x \in\left(E_{2} \backslash P\right) \backslash U$. Sans perte de généralité, supposons que $(0) x^{\prime+}>p$, et donc $(r) x^{\prime-}<1$. Choisissons $y \in P$ tel que $(0) y^{\prime+}=p$ (voir le lemme 4.3). Soit $\gamma \in] 0 ; r\left[\cap A\right.$, choisissons $x_{1}, x_{2} \in \mathcal{F}$ tels que :

(1) $\left.\operatorname{Supp}\left(x_{1}\right)=\right] 0 ; \gamma\left[, \operatorname{Supp}\left(x_{2}\right)=\right] \gamma ; r[$,

(2) $(0) x_{1}^{\prime+}=(0) x^{\prime+},(\gamma) x_{1}^{\prime-}=p^{-1}$,

(3) $(r) x_{2}^{\prime-}=(r) x^{\prime-},(\gamma) x_{2}^{\prime+}=p$.

Alors le centralisateur de $x_{1} x_{2}$ est le produit direct $\left\langle x_{1}\right\rangle \times\left\langle x_{2}\right\rangle$ (voir les lemmes 2.3 et 4.1 ). Choisissons $z \in \mathcal{F}^{\circ}$ tel que $x z=x_{1} x_{2}$. Supposons maintenant que

$$
w_{1}, w_{2} \in C_{\mathcal{F}}(x z)=\left\langle x_{1}\right\rangle \times\left\langle x_{2}\right\rangle .
$$

Alors $(0)\left(y w_{1}\right)^{\prime+} \neq 1$ et $(0)\left(y w_{2}\right)^{\prime+} \neq 1$. Supposons que $y w_{1}, y w_{2} \notin E_{2}$. Alors $(r)\left(y w_{1}\right)^{\prime-}=(r)\left(y w_{2}\right)^{\prime-}=1$, et donc $(r)\left(w_{1} w_{2}^{-1}\right)^{\prime-}=1$, soit encore $w_{1} w_{2}^{-1} \notin E_{2}$. Donc $U$ est en effet 0 -définissable.

Pour tout $x \in P$,

$$
(0) x^{\prime+}=(r) x^{\prime-} \Leftrightarrow(\forall y \in U)\left(\exists z \in C_{\mathcal{F}}(y)\right)\left(x z, x z^{-1} \notin E_{2}\right) .
$$

Donc, l'ensemble $B$ est 0-définissable.

La $f$-préimage du graphe de l'addition de $\mathbb{N}$ est 0 -définissable comme elle est le graphe de la multiplication modulo $\mathcal{F}^{\circ}$ : pour tous $x, y, z \in B$,

$$
(x) f+(y) f=(z) f \Leftrightarrow x y z^{-1} \in \mathcal{F}^{\circ} .
$$

Il nous reste à montrer que la $f$-préimage du graphe de la divisibilité de $\mathbb{N}$ est 0-définissable. C'est en effet le cas : pour tous $x, y \in B$,

$$
(x) f \mid(y) f \Leftrightarrow\left(\forall z \in \mathcal{F}^{\circ}\right)\left(\exists w \in C_{\mathcal{F}}(x z)\right)\left(y w \in \mathcal{F}^{\circ}\right) .
$$

L'implication « $\Leftarrow »$ ici est la moins évidente. Afin de la démontrer par contraposé, on peut prendre $\gamma \in] 0 ; r\left[\cap A\right.$, choisir $x_{1}, x_{2} \in \mathcal{F}$ tels que :

(1) $\left.\operatorname{Supp}\left(x_{1}\right)=\right] 0 ; \gamma\left[, \operatorname{Supp}\left(x_{2}\right)=\right] \gamma ; r[$,

(2) $(0) x_{1}^{\prime+}=(0) x^{\prime+},(\gamma) x_{1}^{--}=p^{ \pm 1}$,

(3) $(r) x_{2}^{\prime-}=(r) x^{\prime-},(\gamma) x_{2}^{\prime+}=p^{ \pm 1}$,

et choisir $z \in \mathcal{F}^{\circ}$ tel que $x z=x_{1} x_{2}$ (et donc $C_{\mathcal{F}}(x z)=\left\langle x_{1}\right\rangle \times\left\langle x_{2}\right\rangle$ ). 
Le théorème $\mathrm{B}$ est une conséquence de la proposition 5.2.

\section{INDÉCIDABILITÉ}

Dans cette section, nous déduisons de [19, théorème 9] (voir le théorème 6.3 ci-dessous) que la théorie élémentaire de toute structure de signature finie qui interprète l'Arithmétique avec paramètres est héréditairement indécidable.

Dans ce qui suit, les constantes sont traitées comme des fonctions d'arité 0 , et également des symboles de constante sont vus comme un cas particulier de symboles de fonction. Si $\sigma$ est un symbole de relation, de fonction, ou de constante, notons $\operatorname{ar}(\sigma)$ l'arité de $\sigma$.

Disons qu'une relation $R n$-aire sur un ensemble $B$ est compatible avec une relation d'équivalence $E$ sur $B$ si $R$ est induite par une relation (de la même arité) sur $B / E$, c'est-à-dire si l'appartenance à $R$ d'une $n$-uple $\left(b_{1}, \ldots, b_{n}\right)$ ne dépend que des classes de $E$-équivalence de $b_{1}, \ldots, b_{n}$.

Soient $\Sigma$ et $\Gamma$ deux signatures, et soit $N$ une $\Gamma$-structure. Soient $n \in \mathbb{N}, B$ une partie définissable de $N^{n}$, et $E$ une relation d'équivalence sur $B$ aussi définissable dans $N$. Soient $\phi$, $\psi$, et $\xi_{\sigma}$ pour tout $\sigma \in \Sigma$ des $\Gamma$-formules avec des paramètres extraits de $N$ telles que :

(1) $\phi$ définisse $B$,

(2) $\psi$ définisse $E$,

(3) pour tout symbole de relation $\sigma \in \Sigma$, la formule $\xi_{\sigma}$ définisse une relation sur $B$ compatible avec $E$ d'arité $\operatorname{ar}(\sigma) n$, et

(4) pour tout symbole de fonction ou de constante $\sigma \in \Sigma$, la formule $\xi_{\sigma}$ définisse une relation sur $B$ d'arité $(\operatorname{ar}(\sigma)+1) n$ qui est compatible avec $E$ et qui définit le graphe d'une opération sur $B / E$ d'arité $\operatorname{ar}(\sigma)$.

Alors notons $\mathfrak{I n t}_{\Sigma}\left(N, \phi, \psi,\left(\xi_{\sigma}\right)_{\sigma \in \Sigma}\right)$ la $\Sigma$-structure définie sur $B / E$ par la famille $\left(\xi_{\sigma}\right)_{\sigma \in \Sigma}$ au sens naturel.

Remarque 6.1. Dans la même notation, la projection naturelle $p: B \rightarrow B / E$ est une interprétation de $\mathfrak{I n t}_{\Sigma}\left(N, \phi, \psi,\left(\xi_{\sigma}\right)_{\sigma \in \Sigma}\right)$ dans $N$.

Lemme 6.2. Soient $M$ et $N$ deux structures de signatures finies telles que $\operatorname{Th}(M)$ soit héréditairement indécidable, et que $N$ interprète $M$ avec paramètres. Alors $\operatorname{Th}(N)$ est héréditairement indécidable aussi.

Démonstration. Notons $\Sigma$ la signature de $M$ et $\Gamma$ la signature de $N$.

Il suffit de considérer le cas où $\Sigma$ ne contient pas de symboles de fonction, ni de symboles de constante. En effet, soit $\Sigma^{\prime}$ la signature obtenue à partir de $\Sigma$ en remplaçant tout symbole de fonction $n$-aire $f(n \geq 0)$ par un symbole de relation $(n+1)$-aire $f^{\prime}$. Pour toute $\Sigma$-structure $M$, notons $M^{\prime}$ la $\Sigma^{\prime}$-structure sur l'ensemble sous-jacent de $M$ où tout nouveau symbole de relation de $\Sigma^{\prime}$ est interprété par le graphe de la fonction dans $M$ nommée par le symbole de $\Sigma$ correspondant, et tous les autres symboles de $\Sigma^{\prime}$ sont interprétés dans $M^{\prime}$ exactement comme dans $M$. Pour toute $\Sigma$-théorie $S$, notons $S^{\prime}$ la $\Sigma^{\prime}$-théorie de la classe $\left\{M^{\prime} \mid M \models S\right\}$. Il est facile de voir que :

(1) la (classe-)application $M \mapsto M^{\prime}$, où $M$ est un $\Sigma$-modèle de $S$, est une (classe-) bijection entre $\operatorname{Mod}_{\Sigma} S$ et $\operatorname{Mod}_{\Sigma^{\prime}} S^{\prime}$ pour toute $\Sigma$-théorie $S$, 
(2) si $O_{\Sigma}$ dénote la $\Sigma$-théorie minimale, alors l'application $S \mapsto S^{\prime}$ de $\Sigma$ théories vers $\Sigma^{\prime}$-théories est une bijection entre toutes les $\Sigma$-théories et toutes les $\Sigma^{\prime}$-théories contenant $O_{\Sigma}^{\prime}\left(O_{\Sigma}^{\prime}\right.$ exprime tout simplement que les nouveaux symboles de relation de $\Sigma^{\prime}$ s'interprètent par des graphes de fonctions),

(3) pour toute $\Sigma$-structure $M$, un ensemble est 0-définissable dans $M$ si et seulement si il l'est dans $M^{\prime}$, ou autrement dit, $\mathrm{id}_{M}$ est une 0-interprétation de $M$ dans $M^{\prime}$ et de $M^{\prime}$ dans $M$.

Il est facile de fournir un algorithme qui convertit tout $\Sigma$-énoncé $\phi$ en un $\Sigma^{\prime}$-énoncé $\psi$ tel que pour toute $\Sigma$-structure $M, M \models \phi$ si et seulement si $M^{\prime} \mid=\psi$, et il est également facile de fournir un algorithme qui convertit tout $\Sigma^{\prime}$-énoncé en un $\Sigma$-énoncé équivalent dans le même sens. En conséquence, pour toute $\Sigma$-théorie $S, S^{\prime}$ est essentiellement indécidable si et seulement si $S$ l'est. Donc nous supposons sans perte de généralité que $\Sigma$ ne contienne que des symboles de relation.

Soit $(n, f)$ une interprétation de $M$ dans $N$. Soit $a_{1}, \ldots, a_{m}$ une suite des paramètres extraits de $N$ suffisante pour définir l'ensemble de départ et le noyau de $f$ et la $f$-préimage du graphe de toute relation de $M$ ( $\Sigma$ est finie) ; notons $\bar{a}=\left(a_{1}, \ldots, a_{m}\right)$. Soient $x_{1}, \ldots, x_{m}, y_{1}, \ldots, y_{n}, y_{11}, \ldots, y_{1 n}$, $y_{21}, \ldots, y_{2 n}, \ldots$ des variables différentes, et notons $\bar{x}=\left(x_{1}, \ldots, x_{m}\right), \bar{y}=$ $\left(y_{1}, \ldots, y_{n}\right), \bar{y}_{1}=\left(y_{11}, \ldots, y_{1 n}\right)$, et ainsi de suite. Soient $\phi=\phi(\bar{x}, \bar{y}), \psi=$ $\psi\left(\bar{x}, \bar{y}_{1}, \bar{y}_{2}\right)$, et $\xi_{\sigma}=\xi_{\sigma}\left(\bar{x}, \bar{y}_{1}, \ldots, \bar{y}_{k}\right)$ pour tout $\sigma \in \Sigma$ d'arité $k$ des $\Gamma$-formules telles que :

(1) $\phi(\bar{a}, \bar{y})$ définisse l'ensemble de départ de $f$ (qui est une partie de $\left.N^{n}\right)$,

(2) $\psi\left(\bar{a}, \bar{y}_{1}, \bar{y}_{2}\right)$ définisse le noyau de $f$ (qui est une relation d'équivalence sur l'ensemble de départ),

(3) pour tout symbole $\sigma \in \Sigma$, la formule $\xi_{\sigma}\left(\bar{a}, \bar{y}_{1}, \ldots, \bar{y}_{\operatorname{ar}(\sigma)}\right)$ définisse la $f$-préimage du graphe de la relation de $M$ nommée par $\sigma$.

Remarquons que la bijection induite par $f$ entre le quotient de son ensemble de départ par son noyau et son image est un isomorphisme

$$
\mathfrak{I n t}_{\Sigma}\left(N, \phi(\bar{a}, \bar{y}), \psi\left(\bar{a}, \bar{y}_{1}, \bar{y}_{2}\right),\left(\xi_{\sigma}\left(\bar{a}, \bar{y}_{1}, \ldots, \bar{y}_{\operatorname{ar}(\sigma)}\right)\right)_{\sigma \in \Sigma}\right) \stackrel{\cong}{\rightrightarrows} M .
$$

Dans ce qui suit, soit $\bar{c}=\left(c_{1}, \ldots, c_{m}\right)$ une suite de nouveaux symboles de constante. Écrivons $(\Gamma, \bar{c})$ pour noter la signature obtenue à partir de $\Gamma$ en rajoutant $c_{1}, \ldots, c_{m}$ (comme des symboles de constante).

Soit $\tau=\tau(\bar{x})$ une $\Gamma$-formule telle que la $(\Gamma, \bar{c})$-énoncé $\tau(\bar{c})$ exprime que :

(1) $\psi\left(\bar{c}, \bar{y}_{1}, \bar{y}_{2}\right)$ définit une relation d'équivalence sur l'ensemble défini $\operatorname{par} \phi(\bar{c}, \bar{y})$,

(2) pour tout symbole de relation $\sigma \in \Sigma$, la formule $\xi_{\sigma}\left(\bar{c}, \bar{y}_{1}, \ldots, \bar{y}_{\operatorname{ar}(\sigma)}\right)$ définit une relation sur l'ensemble défini par $\phi(\bar{c}, \bar{y})$ qui est compatible avec la relation d'équivalence définie par $\psi\left(\bar{c}, \bar{y}_{1}, \bar{y}_{2}\right)$.

Clairement tout cela s'exprime au premier ordre. ${ }^{7}$ Notons que

$$
N \models \tau(\bar{a}) .
$$

\footnotetext{
${ }^{7}$ On peut prendre comme $\tau(\bar{c})$ la conjonction des conditions d'admissibilité au sens de [15, section 5.3].
} 
Choisissons une application $t$ récursive (i.e. calculable par un algorithme) de l'ensemble des $\Sigma$-énoncés vers l'ensemble des $\Gamma$-formules avec toutes ses variables libres comprises parmi $x_{1}, \ldots, x_{m}$ telle que pour tout $\Sigma$-énoncé $\alpha$ et toute $(\Gamma, \bar{c})$-structure $L$ telle que $L=\tau(\bar{c})$, $\left(L=\alpha^{t}(\bar{c})\right) \Leftrightarrow\left(\mathfrak{I n t}_{\Sigma}\left(L, \phi(\bar{c}, \bar{y}), \psi\left(\bar{c}, \bar{y}_{1}, \bar{y}_{2}\right),\left(\xi_{\sigma}\left(\bar{c}, \bar{y}_{1}, \ldots, \bar{y}_{\operatorname{ar}(\sigma)}\right)\right)_{\sigma \in \Sigma}\right) \models \alpha\right)$. Il est facile de construire une telle $t$ qui utilise la formule $\phi$ pour relativiser les quanteurs, la formule $\psi$ pour remplacer $\ulcorner=\urcorner$, et la formule $\xi_{\sigma}$ pour remplacer chaque $\sigma \in \Sigma .^{8}$ Voici un exemple, où $\sigma$ est un symbole de relation binaire :

$$
\begin{gathered}
\alpha=\left\ulcorner\left(\forall y_{1}, y_{2}, y_{3}\right)\left(\sigma\left(y_{1}, y_{2}\right) \wedge \sigma\left(y_{1}, y_{3}\right) \rightarrow y_{2}=y_{3}\right)\right\urcorner, \\
\alpha^{t}(\bar{x})=\left\ulcorner( \forall \overline { y } _ { 1 } , \overline { y } _ { 2 } , \overline { y } _ { 3 } ) \left(\phi\left(\bar{x}, \bar{y}_{1}\right) \wedge \phi\left(\bar{x}, \bar{y}_{2}\right) \wedge \phi\left(\bar{x}, \bar{y}_{3}\right)\right.\right. \\
\left.\left.\rightarrow\left(\xi_{\sigma}\left(\bar{x}, \bar{y}_{1}, \bar{y}_{2}\right) \wedge \xi_{\sigma}\left(\bar{x}, \bar{y}_{1}, \bar{y}_{3}\right) \rightarrow \psi\left(\bar{x}, \bar{y}_{2}, \bar{y}_{3}\right)\right)\right)\right\urcorner .
\end{gathered}
$$

(Comme à l'accoutumée, nous ne montrons pas toutes les parenthèses; elles devraient être ajoutées selon les règles standards.)

Notons les propriétés suivantes de $t$ :

(1) pour tout $\Sigma$-énoncé $\alpha$,

$$
(M \models \alpha) \Leftrightarrow\left(N \models \alpha^{t}(\bar{a})\right) ;
$$

(2) pour tout $\Sigma$-énoncé $\alpha$,

$$
\left(\vdash_{\Sigma} \alpha\right) \Rightarrow\left(\tau(\bar{c}) \vdash_{(\Gamma, \bar{c})} \alpha^{t}(c)\right) ;
$$

(3) pour tous $\Sigma$-énoncés $\alpha$ et $\beta$,

$$
\begin{aligned}
& \tau(\bar{c}) \vdash_{(\Gamma, \bar{c})}(\alpha \wedge \beta)^{t}(c) \leftrightarrow \alpha^{t}(c) \wedge \beta^{t}(c), \\
& \tau(\bar{c}) \vdash_{(\Gamma, \bar{c})}(\neg \alpha)^{t}(c) \leftrightarrow \neg \alpha^{t}(c),
\end{aligned}
$$

et de même pour les autres opérations booléennes;

(4) pour toute $(\Gamma, \bar{c})$-théorie $T$ telle que $T \vdash_{(\Gamma, \bar{c})} \tau(\bar{c})$, l'ensemble

$$
\left\{\Sigma \text {-énoncé } \alpha \mid T \vdash_{(\Gamma, \bar{c})} \alpha^{t}(\bar{c})\right\}
$$

est une $\Sigma$-théorie.

Supposons maintenant que $\operatorname{Th}(N)$ ne soit pas héréditairement indécidable. Alors soit $T$ une $\Gamma$-sous-théorie décidable de $\operatorname{Th}(N)$. Soit $S$ l'ensemble de toutes les $\Gamma$-formules $\alpha(\bar{x})$ telles que

$$
T \vdash_{\Gamma}(\forall \bar{x})(\tau(\bar{x}) \rightarrow \alpha(\bar{x})) .
$$

Alors $\tau \in S, N=\alpha(\bar{a})$ pour toute $\alpha \in S, S$ est un ensemble récursif (décidable), et $\{\alpha(\bar{c}) \mid \alpha(\bar{x}) \in S\}$ est une $(\Gamma, \bar{c})$-théorie. Soit $U$ la préimage de $S$ sous $t$. Alors $U \subset \operatorname{Th}(M), U$ est une $\Sigma$-théorie, et $U$ est décidable contrairement à l'indécidabilité héréditaire de $\operatorname{Th}(M)$.

Théorème 6.3 (Mostowski, Tarski, [20]). La théorie élémentaire de l'Arithmétique $(\mathbb{N},+, \times)$ a une sous-théorie essentiellement indécidable et finiment axiomatisée.

\footnotetext{
${ }^{8}$ Dans [15, section 5.3] une telle $t$ est dite une application de réduction.
} 
Pour une preuve amélorée de ce fait, voir [19, théorème 9].

Proposition 6.4. Soit $M$ une structure de signature finie qui interprète l'Arithmétique $(\mathbb{N},+, \times)$ avec paramètres. Alors $\operatorname{Th}(M)$ est héréditairement indécidable.

Démonstration. C'est un corollaire du théorème $\underline{6.3}$ et des lemmes $\underline{2.13}$ et $\underline{6.2}$.

Le théorème $\mathrm{C}$ (voir l'introduction) résulte maintenant du théorème $\mathrm{A}$ et de la proposition 6.4 .

\section{Questions Ouvertes}

Nous concluons avec deux questions qui, à notre connaissance, sont ouvertes.

Le groupe $F$ de Thompson est définissable dans $T{ }^{9}$ Par contre, il n'est pas connu aux auteurs si $F$ est définissable dans $V$.

Question 1. Le groupe $F$ de Thompson, est-il définissable dans $V$ avec paramètres?

Nous avons déjà démontré que l'Arithmétique s'interprète dans $F$. Par ailleurs, $F$ s'interprète dans l'Arithmétique parce que le problème des mots $\mathrm{y}$ est décidable. (Le théorème de Matiyasevich, voir [18] ou [9, théorème 8.1], implique que tout sous-ensemble récursif ou récursivement énumérable de $\mathbb{N}^{n}, n \in \mathbb{N}$, est définissable dans l'Arithmétique ; ainsi tout codage raisonnable des éléments de $F$ par des entiers positifs donne une interprétation de $F$ dans l'Arithmétique.) Pourtant, même si une structure interprète l'Arithmétique et que réciproquement elle est interprétée dans celle-ci, il n'est pas nécessaire que les deux structures soient bi-interprétables (voir [17, théorème 6] ou [21, théorème 7.16]), d'où la question.

Question 2. L'Arithmétique et le groupe $F$, sont-ils bi-interprétables parmi eux avec paramètres?

Disons que une structure $S$ est catégoriquement finiment axiomatisée dans une classe $C$ de structures de la même signature si $S \in C$ et qu'il existe un énoncé $\phi$ du premier ordre tel que $S \models \phi$ et que toute structure dans $C$ qui satisfait $\phi$ soit isomorphe à $S .{ }^{10}$ Selon Anatole Khélif [17], la biinterprétabilité avec l'Arithmétique peut être utilisée pour établir l'axiomatisation finie catégorique dans des classes de structures de signatures finie et de type fini. Thomas Scanlon [31] a récemment établi la bi-interprétabilité entre l'Arithmétique et tout corps commutatif de type fini et a utilisé cela pour démontrer que tout tel corps est catégoriquement finiment axiomatisé dans la classe de tous corps commutatifs de type fini, et ainsi que la conjecture de Pop est vrai :

\footnotetext{
${ }^{9}$ Nous n'allons pas montrer cela ici.

${ }^{10}$ Dans le cas où la classe $C$ est formée de toutes les structures de type fini d'une certaine classe, André Nies [21] et Anatole Khélif [17] ont utilisé le terme « quasi finiment axiomatisé » dans plus ou moins le même sens que nous utilisons " catégoriquement finiment axiomatisé ». Cependant les définitions dans [21] et [17] ne sont pas précises parce que la propriété d'être de type fini n'est pas interne et dépend de la classe dans laquelle une structure donnée est considérée.
} 
deux corps commutatifs de type fini sont élémentairement équivalents si et seulement si ils sont isomorphes.

André Nies a soulevé la question s'il existe un groupe simple de type fini catégoriquement finiment axiomatisé parmi tous les groupes simples de type fini, voir [21, question 7.8].

\section{Appendice}

Ici nous démontrons que tout élément du sous-groupe dérivé de $\mathcal{F}$ est le produit de deux commutateurs, et donc que $[\mathcal{F}, \mathcal{F}]$ est 0 -définissable dans $\mathcal{F}$. La preuve de ce fait, que par ailleurs nous n'avons pas utilisé dans ce travail, est connue aux spécialistes. Cependant, ce fait est étroitement lié à la structure définissable des groupes à l'étude, et apparemment il ne paraît nulle part ailleurs dans la littérature. Pour le groupe $F$ de Thompson, ce résultat probablement fait partie du folklore; nous avons appris sa preuve de Matthew Brin, qui avait légèrement modifié l'argument de Keith Dennis et de Leonid Vaserstein [11, proposition 1(c)]. Notre argument n'est qu'une généralisation triviale.

Comme dans la preuve de la proposition 5.2, notons

$$
\mathcal{F}^{\circ}=\left\{x \in \mathcal{F} \mid(0) x^{+}=(r) x^{\prime-}=1\right\} .
$$

Proposition 8.1. Tout élément de $[\mathcal{F}, \mathcal{F}]$ est le produit de deux commutateurs dans $\mathcal{F}$, voire dans $\mathcal{F}^{\circ}$ :

$$
[\mathcal{F}, \mathcal{F}]=\left\{\left[x_{1}, x_{2}\right]\left[x_{3}, x_{4}\right] \mid x_{1}, x_{2}, x_{3}, x_{4} \in \mathcal{F}^{\circ}\right\} .
$$

Démonstration. Tout d'abord rappelons que $[\mathcal{F}, \mathcal{F}] \subset \mathcal{F}^{\circ}$ (voir le lemme 4.1).

Nous nous donnons maintenant deux éléments quelconques $x, y \in \mathcal{F}$ et leur commutateur $c=[x, y]$. Soient $\alpha_{1}, \alpha_{2}, \beta_{1}, \beta_{2} \in A$ tels que $0<\alpha_{2}<\alpha_{1}<$ $\beta_{1}<\beta_{2}<r$ et que $\left.\operatorname{Supp}(c) \subset\right] \alpha_{1} ; \beta_{1}[$. Alors il existe un endomorphisme $h: \mathcal{F} \rightarrow \mathcal{F}_{] \alpha_{2} ; \beta_{2}[}$ tel que $h$ soit l'identité sur $\mathcal{F}_{] \alpha_{1} ; \beta_{1}[}$. En effet, soit $s:[0 ; r[\rightarrow$ $\left[0 ; r\left[\right.\right.$ une application qui est l'identité sur $\left[\alpha_{1} ; \beta_{1}\right]$ et qui est affine sur $\left[0 ; \alpha_{1}\right]$ et sur $\left[\beta_{1} ; r[\right.$ avec une pente $p \ll 1, p \in \Lambda$, alors on peut prendre comme $h$ la conjugaison par $s$ composée avec le plongement naturel des permutations de l'intervalle $[(0) s ;(r) s[$ dans les permutations de $[0 ; r[$ (cet $h$ est même injective). Si $h$ est un tel endomorphisme, $(x) h=x^{\prime}$, et que $(y) h=y^{\prime}$, alors

$$
\left.c=(c) h=\left[x^{\prime}, y^{\prime}\right] \quad \text { et } \operatorname{Supp}\left(x^{\prime}\right) \cup \operatorname{Supp}\left(y^{\prime}\right) \subset\right] \alpha_{2} ; \beta_{2}[.
$$

Cela entraîne deux conséquences d'intérêt pour nous :

(1) si $x, y \in \mathcal{F}$, alors il existe $x^{\prime}, y^{\prime} \in \mathcal{F}^{\circ}$ tels que $[x, y]=\left[x^{\prime}, y^{\prime}\right]$;

(2) si $c_{1}, c_{2}, \ldots, c_{n}$ sont des commutateurs dans $\mathcal{F}$, et $I_{1}, I_{2}, \ldots, I_{n}$ sont des sous-intervalles deux-à-deux disjoints fermés de $[0 ; r[$, et tels que $\operatorname{Supp}\left(c_{i}\right) \subset I_{i}$ pour tout $i=1, \ldots, n$, alors le produit $c_{1} c_{2} \cdots c_{n}$ est un commutateur aussi.

Soient maintenant $c_{1}, c_{2}$, et $c_{3}$ trois commutateurs arbitraires dans $\mathcal{F}$. Choisissons $\alpha, \beta \in A \cap] 0 ; r[$ tels que

$$
\left.\operatorname{Supp}\left(c_{1}\right) \cup \operatorname{Supp}\left(c_{2}\right) \cup \operatorname{Supp}\left(c_{3}\right) \subset\right] \alpha ; \beta[.
$$


Choisissons $b \in \mathcal{F}$ tel que $(\alpha) b>\beta$. Alors $0<(\beta) b^{-1}<\alpha<\beta<(\alpha) b<r$. Comme

$$
\left.\operatorname{Supp}\left(c_{2}^{b}\right) \subset\right](\alpha) b ; r\left[\quad \text { et } \operatorname{Supp}\left(c_{3}^{b^{-1}}\right) \subset\right] 0 ;(\beta) b^{-1}[,
$$

le produit $c_{1} c_{2}^{b} c_{3}^{b^{-1}}$ est un commutateur. ${ }^{11}$ D'où

$$
c_{1} c_{2} c_{3}=c_{1} c_{2}^{b} c_{3}^{b^{-1}}\left(\left(c_{3}^{-1}\right)^{b^{-1}} c_{2}\right)\left(\left(c_{2}^{-1}\right)^{b} c_{3}\right)=\left(c_{1} c_{2}^{b} c_{3}^{b^{-1}}\right)\left[c_{2}^{-1} c_{3}^{b^{-1}}, b\right]
$$

est le produit de deux commutateurs.

\section{RÉFÉRENCES}

[1] Gisela Ahlbrandt et Martin Ziegler, Quasi finitely axiomatizable totally categorical theories, Ann. Pure Appl. Logic 30 (1986), no. 1, 63-82 (anglais).

[2] Valery G. Bardakov et Vladimir A. Tolstykh, Interpreting the arithmetic in Thompson's group F, J. Pure Appl. Algebra 211 (2007), no. 3, 633-637 (anglais). Prépublication : arXiv:math/0701748.

[3] James M. Belk et Kenneth S. Brown, Forest diagrams for elements of Thompson's group F, Internat. J. Algebra Comput. 15 (2005), no. 5-6, 815-850 (anglais).

[4] Robert Bieri et Ralph Strebel, On groups of PL-homeomorphisms of the real line, Math. Sem. der Univ. Frankfurt, notes non publiées, 1985.

[5] Collin Bleak, Alison Gordon, Garrett Graham, Jacob Hughes, Francesco Matucci, Hannah Newfield-Plunkett, et Eugenia Sapir, Using dynamics to analyze centralizers in the generalized Higman-Thompson groups $V_{n}$, prépublication en version incomplète (anglais).

[6] Matthew G. Brin et Craig. C. Squier, Presentations, conjugacy, roots, and centralizers in groups of piecewise linear homeomorphisms of the real line, Comm. Algebra, 29 (2001), no. 10, 4557-4596 (anglais).

[7] Kenneth S. Brown, Finiteness properties of groups, J. Pure Appl. Algebra 44 (1987), no. $1-3,45-75$ (anglais).

[8] James W. Cannon, William J. Floyd, et Walter R. Parry, Introductory notes on Richard Thompson's groups, Enseign. Math. (2) 42 (1996), no. 3-4, 215-256 (anglais). Prépublication : http://www.geom.uiuc.edu/docs/preprints/lib/GCG63/ thompson.ps.

[9] Martin Davis, Hilbert's tenth problem is unsolvable, Amer. Math. Monthly 80 (1973), 233-269 (anglais).

[10] Françoise Delon et Patrick Simonetta, Undecidable wreath products and skew power series fields, J. Symbolic Logic 63 (1998), no. 1, 237-246 (anglais).

[11] R. Keith Dennis et Leonid N. Vaserstein, Commutators in linear groups, $K$-Theory 2 (1989), no. 6, 761-767 (anglais).

[12] Yuri L. Ershov, Problemy razreshimosti i konstruktivnye modeli [Problèmes de décidabilité et modèles constructifs], Matematicheskaya Logika i Osnovaniya Matematiki [Logique Mathématique et Fondations de Mathématique], Nauka, Moscow, 1980 (russe).

[13] Kurt Gödel, Über formal unentscheidbare Sätze der Principia Mathematica und verwandter Systeme. I [Sur les propositions formellement indécidables des Principia Mathematica et des systèmes apparentés. I], Monatsh. Math. 149 (2006), no. 1, 1-30 (allemand), réimprimé sur Monatsh. Math. Phys. 38 (1931), 173-198, avec une introduction par Sy-David Friedman.

[14] Graham Higman, Finitely presented infinite simple groups, Notes on Pure Mathematics, vol. 8, Australian National University, Canberra, 1974 (anglais).

[15] Wilfrid Hodges, Model theory, Encyclopedia of Mathematics and its Applications, vol. 42, Cambridge University Press, 1993 (anglais).

\footnotetext{
${ }^{11}$ Nous utilisons la notation standard : $x^{y}=y^{-1} x y,[x, y]=x^{-1} y^{-1} x y$.
} 
[16] _ A shorter model theory, Cambridge University Press, 1997 (anglais).

[17] Anatole Khélif, Bi-interprétabilitée et structures QFA : étude de groupes résolubles et des anneaux commutatifs, C. R. Math. Acad. Sci. Paris 345 (2007), no. 2, 59-61.

[18] Yuri V. Matijasevich, Diofantovost' perechislimykh mnozhestv [La nature diophantienne des ensembles énumérables], Dokl. Akad. Nauk SSSR 191 (1970), 279-282 (russe). Traduction anglaise dans Soviet Math. Dokl.

[19] Andrzej Mostowski, Raphael M. Robinson, et Alfred Tarski, Undecidability and essential undecidability in arithmetic, Undecidable theories, Studies in logic and the foundations of mathematics, North-Holland Publishing Co., Amsterdam, 1971, pp. 36-74 (anglais).

[20] Andrzej Mostowski et Alfred Tarski, Undecidability in the arithmetic of integers and in the theory of rings, J. Symbolic Logic 14 (1949), 76 (anglais).

[21] André Nies, Describing groups, Bull. Symbolic Logic 13 (2007), no. 3, 305-339 (anglais).

[22] Gennady A. Noskov, Ob elementarnoj teorii konechno porozhdyennoj pochti razreshimoj gruppy [À propos de la théorie élémentaire d'un groupe virtuellement résoluble de type fini], Izv. Akad. Nauk SSSR Ser. Mat. 47 (1983), no. 3, 498-517 (russe). Traduction anglaise dans Math. USSR Izvestiya.

[23] On the elementary theory of a finitely generated almost solvable group, Math. USSR Izvestiya 22 (1984), no. 3, 465-482 (anglais). Traduit du russe.

[24] Anand Pillay, An introduction to stability theory, Oxford Logic Guides, vol. 8, The Clarendon Press, Oxford University Press, New York, 1983 (anglais).

[25] Bruno Poizat, Cours de théorie des modèles. Une introduction à la logique mathématique contemporaine, Nur al-Mantiq wal-Ma'rifah, Bruno Poizat, Lyon, 1985.

[26] - Groupes stables. Une tentative de conciliation entre la géométrie algébrique et la logique mathématique, Nur al-Mantiq wal-Ma'rifah, vol. 2, Bruno Poizat, Lyon, 1987.

[27] Stable groups, Mathematical Surveys and Monographs, vol. 87, American Mathematical Society, 2001 (anglais). Traduit de l'original français de 1987 par Moses Gabriel Klein.

[28] Mike Y. Prest, Model theory and modules, London Mathematical Society Lecture Note Series, vol. 130, Cambridge University Press, 1988 (anglais).

[29] Raphael M. Robinson, Undecidable rings, Trans. Amer. Math. Soc. 70 (1951), 137159 (anglais).

[30] Philipp Rothmaler, Introduction to model theory, Algebra, Logic and Applications, vol. 15, Gordon and Breach Science Publishers, Amsterdam, 2000 (anglais). Traduit et révisé de l'original allemand de 1995 par l'auteur.

[31] Thomas Scanlon, Infinite finitely generated fields are biinterpretable with $\mathbb{N}$, J. Amer. Math. Soc. 21 (2008), no. 3, 893-908, (anglais).

[32] Zlil Sela, Diophantine geometry over groups VIII: stability, prépublication, arXiv: math/0609096, 2006 (anglais).

[33] Melanie Stein, Groups of piecewise linear homeomorphisms, Trans. Amer. Math. Soc. 332 (1992), no. 2, 477-514 (anglais).

[34] Alfred Tarski, A general method in proofs of undecidability, Undecidable theories, Studies in logic and the foundations of mathematics, North-Holland Publishing Co., Amsterdam, 1971, pp. 1-35 (anglais).

[35] Frank O. Wagner, Stable groups, Handbook of algebra, vol. 2, North-Holland Publishing Co., Amsterdam, 2000 (anglais).

[36] Thompson's Group at 40 Years, Problem list of the workshop held 11-14 Jan., 2004, at American Institute of Mathematics, Palo Alto, California, http://www.aimath. org/WWN/thompsonsgroup/thompsonsgroup.pdf, 2004 (anglais). 
ARITHMÉTIQUE DANS DES GROUPES DE PERMUTATIONS D'UN INTERVALLE 29

Université de Lyon ; Université Lyon 1 ; Institut CAMille Jordan CNRS UMR 5208 ; 43 Boulevard du 11 novembre 1918, F-69622 Villeurbanne Cedex, FRANCE 